\title{
A near-infrared study of the bow shocks within the L1634 protostellar outflow
}

\author{
B. O’Connell ${ }^{1,2}$, M. D. Smith ${ }^{1}$, C. J. Davis ${ }^{3}$, K. W. Hodapp ${ }^{4}$, T. Khanzadyan ${ }^{5}$, and T. Ray ${ }^{6}$ \\ 1 Armagh Observatory, College Hill, Armagh BT61 9DG, Northern Ireland, UK \\ 2 Physics Department, Trinity College Dublin, College Green, Dublin 2, Ireland \\ 3 Joint Astronomy Centre, 660 N. A'ohoku Place, University Park, Hilo, Hawaii 96720, USA \\ ${ }^{4}$ Institute for Astronomy, University of Hawaii, 640 N. A'ohoku Place, University Park, Hilo, Hawaii 96720, USA \\ 5 Max-Planck-Institut für Astronomie, Königstuhl 17, 69117 Heidelberg, Germany \\ ${ }^{6}$ School of Cosmic Physics, Dublin Institute for Advanced Studies, 5 Merrion Square, Dublin 2, Ireland
}

Received 21 October 2003 / Accepted 25 February 2004

\begin{abstract}
The L1634 bright-rimmed globule contains an intriguing arrangement of shock structures: two series of aligned molecular shock waves associated with the Herbig-Haro flows HH 240 and HH 241. We present near-infrared spectroscopy and narrow-band imaging in the $(1,0) \mathrm{S}(1)$ and $(2,1) \mathrm{S}(1)$ emission lines of molecular hydrogen. These observations yield the spatial distributions of both the molecular excitation and velocity, which demonstrate distinct properties for the individual bow shocks. Bow shock models are applied, varying the shock physics, geometry, speed, density and magnetic field properties to fit two prominent bow shocks. The models predict that both bows move at $60^{\circ}$ to the plane of the sky. High magnetic fields and low molecular fractions are implied. The advancing compact bow HH 240C is interpreted as a J-type bow (frozen-in magnetic field) with the flanks in transition to C-type (field diffusion). It is a paraboloidal bow of speed $\sim 42 \mathrm{~km} \mathrm{~s}^{-1}$ entering a medium of quite high density $\left(2 \times 10^{4} \mathrm{~cm}^{-3}\right)$. The following bow HH 240A is faster despite a lower excitation, moving through a lower density medium. We find a C-type bow shock model to fit all the data for HH240A. The favoured bow models are then tested comprehensively against published $\mathrm{H}_{2}$ emission line fluxes and $\mathrm{CO}$ spectroscopy. We conclude that, while the $\mathrm{CO}$ emission originates from cloud gas directly set in motion, the $\mathrm{H}_{2}$ emission is generated from shocks sweeping through an outflow. Also considering optical data, we arrive at a global outflow model involving episodic slow-precessing twin jets.
\end{abstract}

Key words. ISM: jets and outflows - stars: circumstellar matter - infrared: stars - ISM: Herbig-Haro objects

\section{Introduction}

Bipolar outflows are driven from protostars by jets or collimated winds (Bally \& Lada 1983; Bachiller 1996; Richer et al. 2000). The outflows sweep up and shock-heat cloud material in their paths. The shocks are often detected as Herbig-Haro (HH) objects in optical emission lines and as molecular hydrogen flows in the near-infrared provided the outflow has pierced an obscuring parental cloud (Reipurth \& Bally 2001). A large volume of swept up and disturbed gas, however, may still be observable at longer wavelengths through emission in $\mathrm{CO}$ rotational transitions, and other molecules. An excellent example of this is associated with the L1634 cloud (Lee et al. 2000). We present here a detailed study of the HH objects in L1634 which possess a particularly striking morphology. Our aim is to understand the dominant physical and dynamical processes in a protostar-environment interaction.

The outflow is driven from IRAS 05173-0555 (Davis et al. 1997), a protostar with an estimated mass infall rate of $2-8 \times$ $10^{-5} M_{\odot} \mathrm{yr}^{-1}$ (Beltrán et al. 2002), bolometric luminosity

Send offprint requests to: B. O’Connell, e-mail: boc@arm. ac.uk of $17 L_{\odot}$ (Reipurth et al. 1993) and bolometric temperature of $77 \mathrm{~K}$ (Mardones et al. 1997), classifying it as undergoing a transition phase from Class 0 to Class I. In addition to this east-west outflow there is a weaker northwest-southeast outflow driven from IRS 7, 40" to the east (Hodapp \& Ladd 1995; Davis et al. 1997). We adopt a distance of $460 \mathrm{pc}$ to the molecular cloud, located in Orion (Bohigas et al. 1993).

The main outflow appears to terminate in two regions of shocked gas, identified as $\mathrm{HH} 240$ and HH241 at visible wavelengths (Cohen 1980). To the west, HH 240A (RNO 40) possesses a high proper motion directed away from the IRAS source and, to the east, HH 241A (RNO40E) is also identified in optical emission lines (Jones et al. 1984; Bohigas et al. 1993).

The impact regions have been explored in near-infrared $\mathrm{H}_{2}$ emission lines where distinct and resolved structures have been identified, as displayed in Fig. 1. To the west, several well-defined bow shocks are found (especially HH 240A-D) whereas numerous scattered knots are found to the east (HH 241A-D) (Hodapp \& Ladd 1995). The projected extent between the two outer D knots is $6.3^{\prime}$, yielding a size of $8.4 \mathrm{pc}$ 


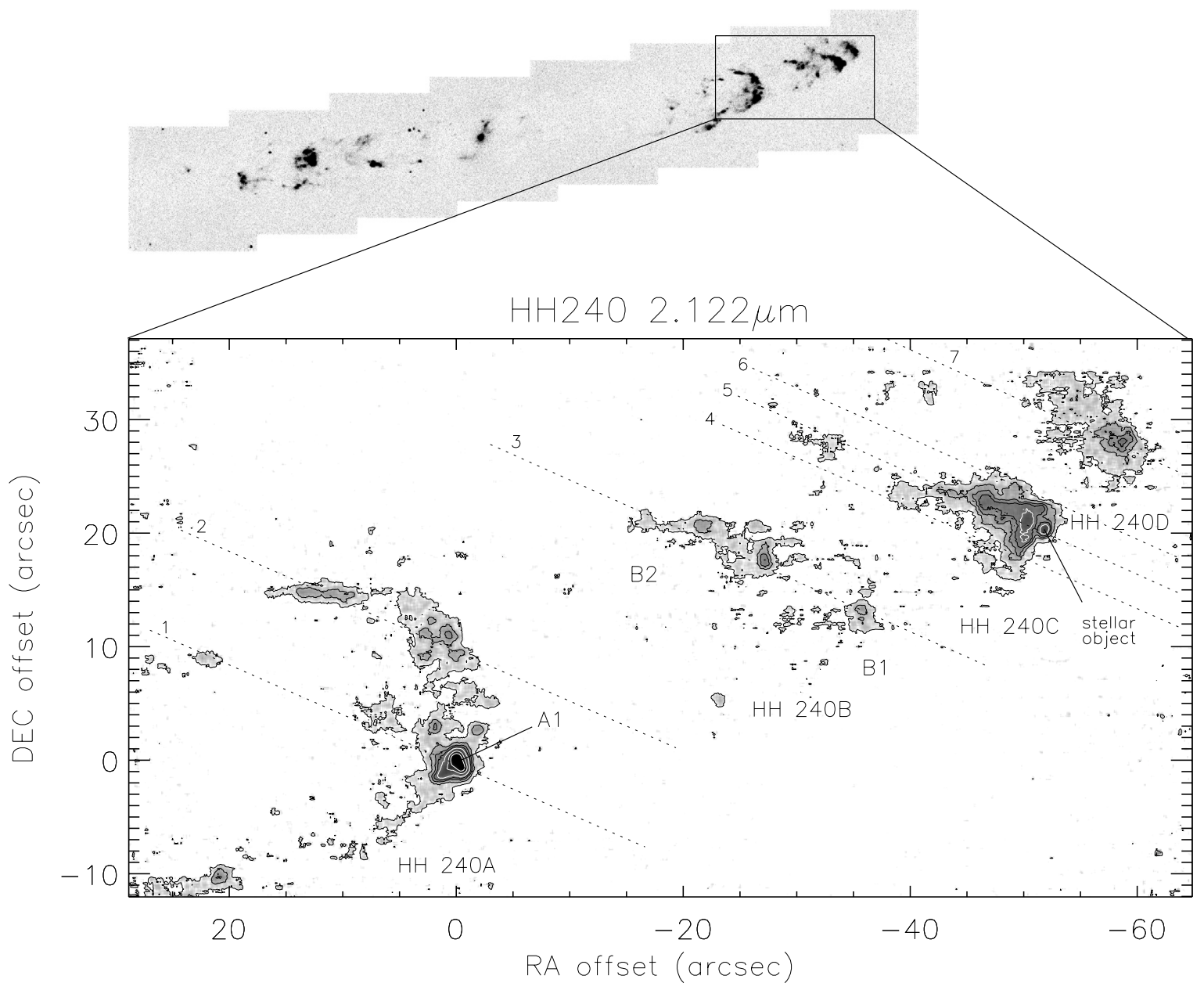

Fig. 1. HH 240 in the $\mathrm{H}_{2}(1,0) \mathrm{S}(1) 2.12 \mu \mathrm{m}$ line. The entire outflow imaged by Davis et al. (1997) is shown above. The contour plot was generated using the same image but smoothed with a 0.'5 FWHM Gaussian. The contour levels are 12, 32, 52, 72 (black), 100, 170, and 240 (white) $\times 10^{-19} \mathrm{~W} \mathrm{~m}^{-2} \operatorname{arcsec}^{-2}$. Offsets are measured from the peak value. The dotted lines represent the positions of echelle spectroscopy slits. The slit numbers correspond to the position velocity diagrams in Fig. 5. Note that the slits here do not represent the actual slit lengths.

at a distance of $460 \mathrm{pc}$. The outer D knots are equidistant from the IRAS source to within measurement error.

The accelerated gas is observed in $\mathrm{CO}$ rotational lines, with most of the blue-shifted CO lying towards the direction of HH 240 (Davis et al. 1997; De Vries et al. 2002). The blue emission curves around the giant HH 240A bow shock with a broad range of radial velocities (including some red-shifted) but there is very little $\mathrm{CO}$ emission beyond this location (Lee et al. 2000). The CO red wing emission to the east takes the form of a hollow shell which ends near the cloud edge (Lee et al. 2000). In the east, however, there is also ample blueshifted CO gas. Lee et al. (2000) favour a jet-driven model with a jet orientation of $30^{\circ}-60^{\circ}$ to the line of sight.

Lynds 1634 is a bright-rimmed cloud, SFO16, associated with Barnard's Loop (Sugitani et al. 1991; De Vries et al. 2002). The $C^{18} \mathrm{O}$ cloud structure suggests an ENE-WSW orientation (De Vries et al. 2002), which may help explain asymmetries in the outflow. There is a small velocity gradient in the cloud, roughly perpendicular to the outflow, which offers the possibility that the outflow lies along the cloud's rotation axis.

In this work, we focus on the remarkable series of $\mathrm{H}_{2}$ bow shocks. Their shapes suggest they are being driven away from the IRAS source by a jet or bullets. Is each bow the result of a distinct protostellar outburst event? Could they form through fluid dynamic instabilities in the jet? Or, are they the consequence of a jet interaction with a non-uniform environment?

High resolution spatial and velocity data permit us to test models for the bow shocks through detailed geometric and physical modelling. We may thus (1) determine the necessary driving power, (2) resolve the debate concerning the shock physics (e.g. C-type or J-type) and (3) limit the speeds of the bow shocks. Recent $1-2.5 \mu \mathrm{m}$ long-slit spectra yield a high molecular excitation for $\mathrm{HH} 240 \mathrm{~A}$, and excitation of atomic lines consistent with a shock speed of $\sim 80 \mathrm{~km} \mathrm{~s}^{-1}$ (Nisini et al. 2002). Our excitation imaging should reveal if the slit spectra are representative of the entire bow shock.

Only a few interstellar bow shocks have been previously imaged in $\mathrm{H}_{2}$ lines from transitions originating above the first vibrational level. One recent such study of $\mathrm{HH} 7$ concluded that C-type shock physics with paraboloidal bow shocks is responsible (Smith et al. 2003b). Here, after presenting (Sect. 2) and discussing (Sect. 3) the new data, we adapt the model developed for that study in order to model these bow shocks employing both C-type and J-type physics (Sect. 4). 
We compare our modelling results with the narrow-band $\mathrm{H}_{2}$ imaging and position-velocity spectroscopy in Sect. 5 as well as with previously published $1-2.5 \mu$ m spectroscopy in Sect. 6 and $\mathrm{CO} J=(1,0)$ imaging and spectroscopy in Sect. 7. In Sect. 8 we discuss our findings in relation to previously published optical data before making several conclusions (Sect. 9) based on this large reservoir of data.

\section{Observations and data reduction}

\subsection{Near-infrared imaging}

On November 12, 2001 near-infrared images of HH 240 were obtained with the Fast Track Imager camera UFTI on the $3.8 \mathrm{~m}$ UK Infrared Telescope (UKIRT), on the summit of Mauna Kea, Hawaii. The camera is fitted with a $1024 \times 1024 \mathrm{HgCdTe}$ Rockwell array which together with the internal optics provides a total field of view of $92^{\prime \prime} .9 \times 92^{\prime \prime} .9$ and a pixel scale of 0. '091 per pixel.

Individual frames were obtained in a jitter pattern and mosaics were constructed in both the $(1,0) \mathrm{S}(1)$ and the $(2,1) \mathrm{S}(1)$ transition lines of $\mathrm{H}_{2}$ using narrow-band filters centered on $\lambda=2.122 \mu \mathrm{m}$ and $\lambda=2.248 \mu \mathrm{m}$ respectively with $F W H M=$ $0.02 \mu \mathrm{m}$. Both filters are situated in the $K$-band where atmospheric transmission is high. The integration time for each individual frame was $100 \mathrm{~s}$. The total on-source exposure times were $15 \mathrm{~min}$ and $90 \mathrm{~min}$ per filter respectively.

The Starlink packages CCDPACK and KAPPA were used to reduce the data. Dark exposures were subtracted from each frame and for each image the set of target frames was combined into a flatfield used to correct for the pixel sensitivity across the array. For each frame the mean count determined from the full array was used to subtract off the background sky level. Guide star tracking was maintained throughout and the telescope registered offsets were used to create the final mosaics due to the lack of field stars.

The standard stars HD 18881 (spectral type A0; $K=$ $7.14 \mathrm{mag}$ ) and HD 43244 (spectral type F0V; $K=6.52 \mathrm{mag}$ ) were observed, at the same airmass as L1634, in both filters in order to flux calibrate the images. The flux from the star in HH 240C was compared in both filters and found to be about equal as would be expected from a continuum source. Seeing throughout the observations was 0.7 . In order to increase the signal to noise ratio without compromising the resolution the mosaics were smoothed by applying a $4 \times 4$ pixel box filter to estimate the mean.

\subsection{Position-velocity $(P-V)$ spectroscopy}

Echelle spectra centred on the $\mathrm{H}_{2}(1,0) \mathrm{S}(1)$ emission line $\left(\lambda_{\mathrm{vac}}=2.1218334 \mu \mathrm{m} ;\right.$ Bragg et al. 1982) were obtained between the 5th and 7th February 1999 (UT) at the UK Infrared Telescope (UKIRT) using the cooled grating spectrometer CGS 4. The instrument was equipped with a $256 \times$ 256 pixel InSb array; the pixel scale was 0 ". $41 \times 0$ ". $90(0$ ". 41 in the dispersion direction). A 2-pixel-wide slit was used, resulting in a velocity resolution of $\sim 15 \mathrm{~km} \mathrm{~s}^{-1}$. The instrumental
Table 1. Photometric results for $\mathrm{HH} 240$.

\begin{tabular}{ccccc}
\hline \hline Object & $\begin{array}{c}\text { Circular } \\
\text { aperture }\end{array}$ & $\begin{array}{c}\mathrm{H}_{2} \\
(1,0) \mathrm{S}(1)^{*}\end{array}$ & $\begin{array}{c}\mathrm{H}_{2} \\
(2,1) \mathrm{S}(1)^{*}\end{array}$ & $\begin{array}{c}\text { Excitation } \\
\text { ratio } \dagger\end{array}$ \\
\hline HH 240A & $30^{\prime \prime}$ & 401 & 43 & $0.11(0.02)$ \\
A1 & $6^{\prime \prime}$ & 157 & 24 & $0.15(0.02)$ \\
HH 240B & $28^{\prime \prime}$ & 149 & 33 & - \\
B1 & $12^{\prime \prime}$ & 51 & 13 & - \\
B2 & $16^{\prime \prime}$ & 91 & 20 & - \\
HH 240C & $18^{\prime \prime}$ & 285 & 42 & $0.15(0.02)$ \\
HH 240D & $14^{\prime \prime}$ & 139 & 25 & $0.18(0.04)$ \\
\hline
\end{tabular}

* Flux in units of $10^{-18} \mathrm{~W} \mathrm{~m}^{-2}$.

The excitation ratio is the integrated $\mathrm{H}_{2}(2,1) \mathrm{S}(1) /(1,0) \mathrm{S}(1)$ flux ratio. The $\mathrm{HH} 240 \mathrm{C}$ flux values were determined after the continuum at $2.104 \mu \mathrm{m}$ was subtracted. Errors determined from the variance in the background are quoted in brackets.

profile in the dispersion direction, measured from Gaussian fits to sky lines, was $\sim 12 \mathrm{~km} \mathrm{~s}^{-1}$.

Data at seven slit positions in $\mathrm{HH} 240$ and five in $\mathrm{HH} 241$ were obtained (see below). A position angle of $63^{\circ}$ was used in $\mathrm{HH} 240$ and $100^{\circ}$ in $\mathrm{HH} 241$. Object-sky-sky-object sequences were repeated at each slit location to build up signal-to-noise, the sky position being offset from the east-west outflow axis. Each spectral image was bias subtracted and flat-fielded. Skysubtracted object frames were then co-added into reduced "groups" (one group frame per slit position). Each reduced group spectral image was subsequently wavelength calibrated using four well-spaced OH sky lines (Oliva \& Origlia 1992) following the steps described by Davis et al. (2001). The absolute velocity calibration, measured from the wavelengths of the $\mathrm{OH}$ lines in calibrated "raw" spectral images, was of the order of $4-5 \mathrm{~km} \mathrm{~s}^{-1}$, although the relative calibration along each slit (from row to row in each spectral image) was much better than this, the optical distortion along columns (sky lines) in the reduced spectral images being much smaller than the pixel scale.

\section{Results}

\subsection{Imaging}

Figure 1 displays the HH 240 outflow. The sequence of principle knots are denoted HH 240 A to D with increasing distance from the IRAS source. The photometric results are presented in Table 1 along with the integrated $\mathrm{H}_{2}(2,1) \mathrm{S}(1) /(1,0) \mathrm{S}(1)$ flux line ratios for each bow feature.

Figure 2 displays the bow shocks, HH 240A and HH 240C, in detail. The close-up images highlight the spatial resolution of the shock structure. Intensity profiles are displayed for horizontal (slit 1) and vertical (slit 2) slits, to facilitate a comparison to models. The slit locations were chosen to highlight the general trend of the flux distribution. The particularly bright region A1 was avoided as this feature dominates the emission to a large extent and is likely the result of the impact of a fast 

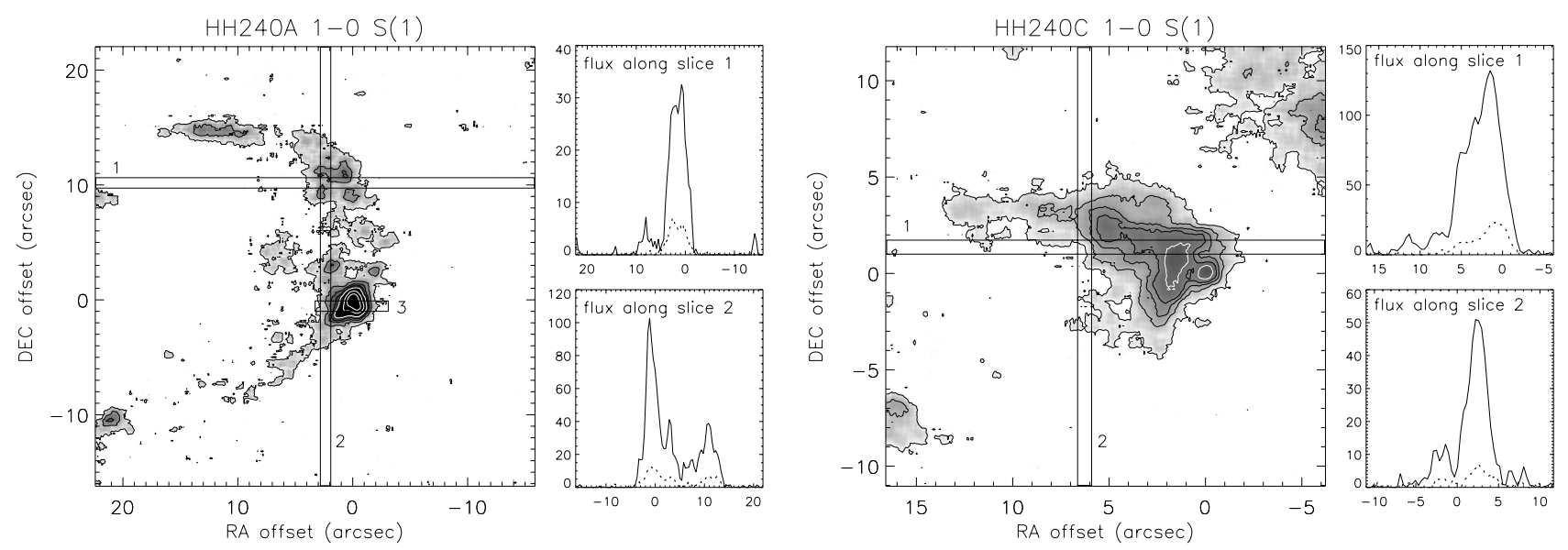

Fig. 2. Individual images of $\mathrm{HH} 240 \mathrm{~A}$ (left) and $\mathrm{HH} 240 \mathrm{C}($ right $)$ in the $\mathrm{H}_{2}(1,0) \mathrm{S}(1)$ line along with vertical and horizontal slices to indicate the flux variation through the bows. The contour levels are 12, 32, 52, 72 (black), 100, 170, and 240 (white) $\times 10^{-19} \mathrm{~W} \mathrm{~m}^{-2}$ arcsec $^{-2}$. The solid line represents the $\mathrm{H}_{2}(1,0) \mathrm{S}(1)$ transition and the dotted line represents the emission from the $\mathrm{H}_{2}(2,1) \mathrm{S}(1)$ transition in units of $10^{-19} \mathrm{~W} \mathrm{~m}^{-2} \operatorname{arcsec}^{-2}$. Offsets are measured from the peak value in each contour image.
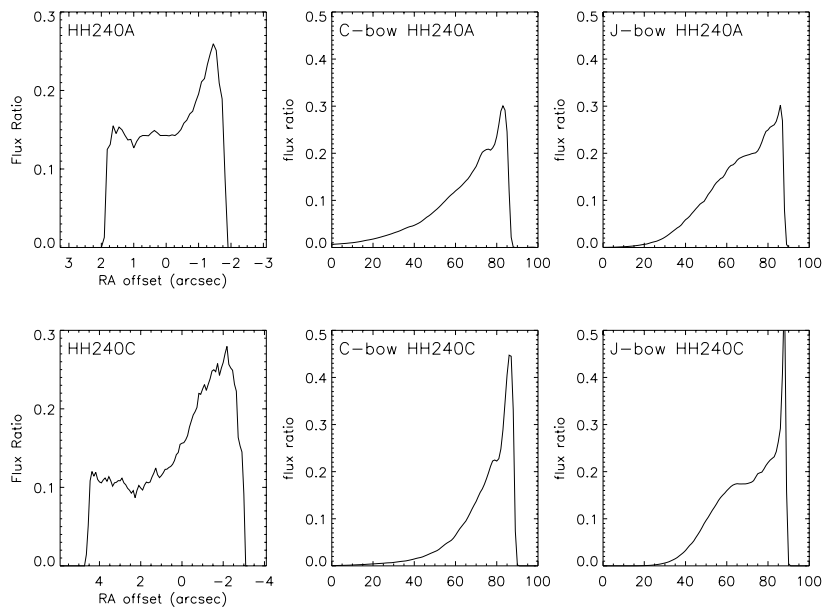

Fig. 3. The $\mathrm{H}_{2}(2,1) /(1,0)$ flux ratio along $\mathrm{HH} 240 \mathrm{~A}$ and $\mathrm{HH} 240 \mathrm{C}$ (left column). The profile for HH240A is along slit 3 in Fig. 2 and the profile for $\mathrm{HH} 240 \mathrm{C}$ is along slit 1 in Fig. 2. For comparison to the models the flux ratio profiles generated in similar locations are shown for C-bow and J-bow models (middle and right column respectively). Note that the profiles for the models cover the whole $x$-scale whereas the observed ratios only cover the portion of the slit which gives measurable quantities.

reverse shock (Mach disk) which is not included in the modelling (See Sect. 8). Along HH 240C the star is avoided in order not to include the continuum flux. Note the narrow peaks as well as the extended distribution. Note also that for the chosen slit locations HH $240 \mathrm{C}$ has a larger horizontal extent but a smaller vertical profile.

Projected onto HH $240 \mathrm{C}$ is a star, evident from the fact that the feature has a sharp point spread function where the flux through both filters is equal: characteristic of a continuum source. This feature has been utilised to align the images in both filters more accurately as there are no other stars within the field of view.

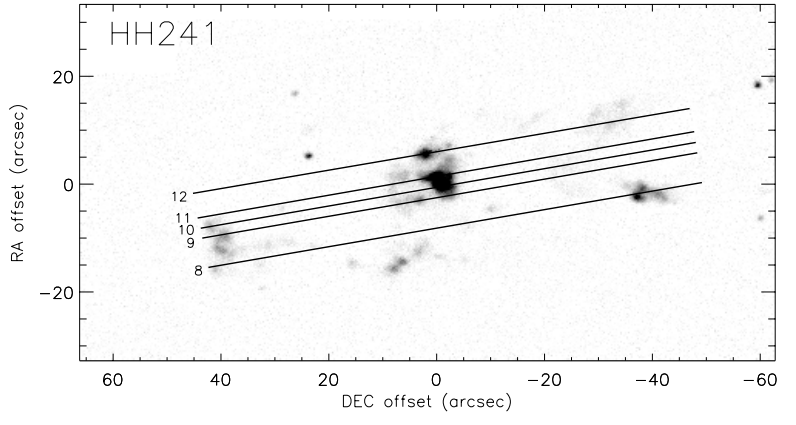

Fig. 4. Spectroscopic slits for HH 241. Each slit is 82'. 8 in length. The slit numbers correspond to the position velocity diagrams in Fig. 5.

A regular asymmetric structure is present: the northern flank of each bow is brighter than the southern edge. This is particularly evident in HH 240C. The bright peak in HH 240A corresponds in position to the optical HH Object, imaged by Bohigas et al. (1993).

Despite the weaker fluxes of the components, the distribution of emission in the $(2,1)$ image (not presented) closely resembles that of the $(1,0)$ image. However, there are some significant variations which are evident in the flux ratio measurements given in Table 1. The variation of this ratio across the bows is presented in Fig. 3 where we have chosen to measure and divide intensity profiles through slits passing through bright and extended emission locations. Both bows possess high excitation peaks at the leading edge followed by low excitation plateaus.

A significant variation in excitation is also present between bows. In particular, HH 240A has a relatively low vibrational excitation. We measure $(2,1) /(1,0) \mathrm{S}(1)$ ratios of 0.11 and 0.15 (with errors of a few per cent) for $\mathrm{HH} 240 \mathrm{~A}$ and $\mathrm{HH} 240 \mathrm{C}$, respectively (Table 1). This result is consistent with the line strengths tabulated by Nisini et al. (2002), on integration along a slit of width $1^{\prime \prime}$. They found $(2,1) /(1,0) \mathrm{S}(1)$ ratios of 0.11 and 0.17 (also with errors of a few per cent) for HH 240A and HH 240C, respectively. 



Fig. 5. Position-velocity diagrams for HH 240 (1-7) and HH 241 (8-12). Labels are according to Figs. 1 and 4 . The location is measured from the western side of the slits in pixels where 1 pixel corresponds to 0.9 and the velocity is relative to the Local Standard of Rest (LSR).

\subsection{Near-infrared echelle spectroscopy}

The positions of 12 slits which pass through interesting features of the shocked $\mathrm{H}_{2}$ emission are marked in Fig. 1 (HH 240) and Fig. 4 (HH 241).

The corresponding position-velocity images are displayed in Fig. 5. The spatial pixel scale of the images is $00^{\prime} 9$ and the angular extension of each image along the $y$-axis is $83^{\prime \prime}$. The systematic velocity of the region, derived from the $\mathrm{N}_{2} \mathrm{H}^{+} J=(1,0)$ hyperfine ensemble, is $+8 \mathrm{~km} \mathrm{~s}^{-1}$ (De Vries et al. 2002).

The most remarkable result is that the $\mathrm{H}_{2}$ emission from $\mathrm{HH} 240$ is blue-shifted relative to the cloud by typically -10 to $-50 \mathrm{~km} \mathrm{~s}^{-1}$. Wide velocity profiles, exceeding $70 \mathrm{~km} \mathrm{~s}^{-1}$ in some locations, are found. However, some emission, in particular from the HH 240A peak in Slit 1, is at the cloud radial velocity. For slits passing through distinct bows (slits 1, 2, 4, 5 and 7) the emission is characterised by a wide peak close to the front followed by emission which grows fainter and trails off at zero velocity. Unlike the $\mathrm{H}_{2}$ bow-shaped structures along the outflow in L1448 (Davis \& Smith 1996), double peaked velocity profiles (indications of bow shock geometry where the $\mathrm{H}_{2}$ emission derives from the front and rear edges of the 3 dimensional bow) are absent except for slit 5 which passes through the compact HH 240C knot. The blue-shifted peak is displaced 2'. 5 back from the red-shifted peak indicating that the bow direction of motion is inclined to the line of sight with the HH $240 \mathrm{C}$ bow directed toward the observer.

The obvious difference between HH 241 and HH 240 is that the $\mathrm{H}_{2}$ emission is almost exclusively red-shifted in all the HH 241 slits. Slits 8 to 12 reveal emission which is on average red-shifted by $26 \mathrm{~km} \mathrm{~s}^{-1}$. The HH 241 outflow is clearly directed away from the observer.

Interestingly, the velocity profiles are narrower in $\mathrm{HH} 241$. They possess an average width of $\sim 45 \mathrm{~km} \mathrm{~s}^{-1}$. This would be expected if we are observing individual shock fronts in $\mathrm{HH} 241$ 
Table 2. Model parameters derived to fit the bow images with C-type shocks. Throughout, we take $n=n(\mathrm{H})+2 n\left(\mathrm{H}_{2}\right)$ as the hydrogen nucleon density.

\begin{tabular}{lll}
\hline \hline Parameter & HH 240A & HH 240C \\
\hline Size, $L_{\text {bow }}$ & $1.7 \times 10^{16} \mathrm{~cm}$ & $1.3 \times 10^{16} \mathrm{~cm}$ \\
(1) H density, $n$ & $2.5 \times 10^{3} \mathrm{~cm}^{-3}$ & $7.0 \times 10^{3} \mathrm{~cm}^{-3}$ \\
(2) Molecular fraction & 0.2 & 0.2 \\
(3) Alfvén speed, $v_{\mathrm{A}}$ & $5 \mathrm{~km} \mathrm{~s}^{-1}$ & $5 \mathrm{~km} \mathrm{~s}^{-1}$ \\
Magnetic field & $135 \mu \mathrm{G}$ & $226 \mu \mathrm{G}$ \\
Ion fraction, $\chi$ & $1 \times 10^{-5}$ & $5 \times 10^{-5}$ \\
(4) Bow velocity, $v_{\text {bow }}$ & $70 \mathrm{~km} \mathrm{~s}^{-1}$ & $50 \mathrm{~km} \mathrm{~s}^{-1}$ \\
(5) Angle to l.o.s. & $60^{\circ}$ & $60^{\circ}$ \\
(6) $s$ parameter & 2.35 & 1.9 \\
(7) Field angle, $\mu$ & $30^{\circ}$ & $60^{\circ}$ \\
\hline
\end{tabular}

rather than superimposed near and far sides of bow shocks. It would still be unclear, however, why the material is exclusively deflected away from the observer. A more detailed interpretation is needed. In Sect. 5, we will model the profiles in terms of bow-shaped shock geometries.

\section{Analysis}

\subsection{Background}

At first sight, all the position-velocity diagrams display features which can be qualitatively attributed to emission resulting from bow-shocked molecular material. The bows are moving (1) sufficiently fast so that $\mathrm{H}_{2}$ is dissociated near the apex and (2) at an angle out of the plane of the sky so that emission from the rear and front of the bow shock surface are projected onto different locations. The HH 240 bows are moving towards us so that the rear sides of the bows give rise to the leading emission when projected onto the sky. Therefore, slits through the front of the bow generate emission which is relatively red-shifted at the front, with a following blue-shifted peak. Moving along the slit into the bow tail, the spread in velocities becomes narrower and the emission intensity fainter. Detailed modelling is presented in Sect. 5.

To model the flux, excitation and velocity information, we employ the bow model described by Smith et al. (2003b). This model assumes a three dimensional geometry for a bow surface with a form

$Z / L_{\text {bow }}=(1 / s)\left(R / L_{\text {bow }}\right)^{s}$

in cylindrical coordinates $(Z, R)$ where the constant $s$ is the shape parameter and $L_{\text {bow }}$ fixes the bow size. We cut up the the bow surface into an extremely large number of planar shocks, each with a particular magnetic field direction relative to the surface normal and orientation relative to the observer, as illustrated in Fig. 6. We integrate through each steady planar shock, taking into account the cooling length and deceleration to determine the flux contribution to a zone in a 3D image-velocity data cube. The planar shock analysis is only accurate if the cooling

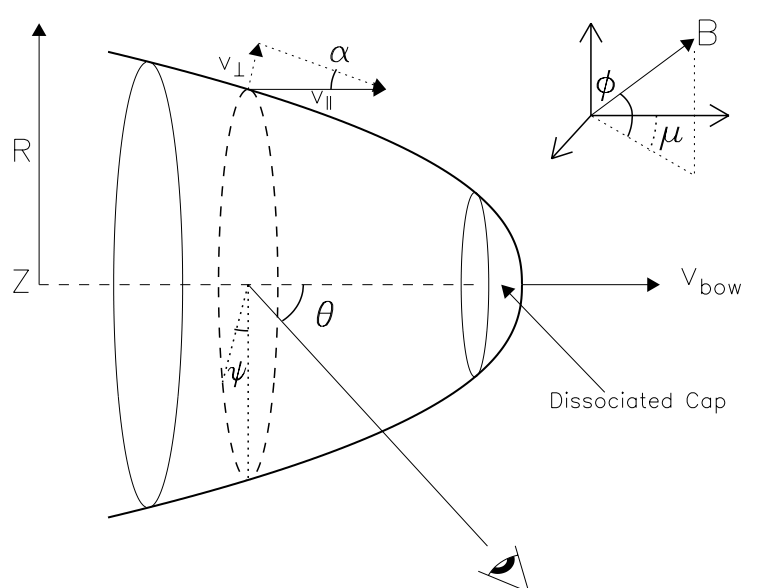

Fig. 6. The geometry employed by the bow-shock model, moving at velocity $V_{\text {bow }}$ whose surface represents a gradation of velocities transverse to the shock surface. The angle to the line of sight $\theta$ and magnetic field direction are parameters which must be chosen.

zone which we are interested in modelling is considerably less than the bow's radius of curvature. This is plausible for HH 240 since the shocks appear thin in $(1,0) \mathrm{S}(1)$ emission.

Bow models can be generated which reflect both C-type (Continuous) and J-type (Jump) shock physics. In the former, the magnetic field has the effect of cushioning the shock through ambipolar diffusion which allows molecules to survive at higher velocities. In the latter, the flow variables experience a discontinuous abrupt shock which dissociates molecules at a lower shock velocity.

To initiate the modelling, at least one of the parameters needs to be estimated; we begin with the density and iterate the fitting of all parameters until a unique fit is reached which optimally reproduces the observed data. A reddening factor, $E_{J-K}$, can be determined from the two brightest [Fe II] forbidden transition lines at $1.257 \mu \mathrm{m}$ and $1.644 \mu \mathrm{m}$ which both originate from the same upper level. Using this method and adopting the Rieke \& Lebofsky (1985) extinction law, Nisini et al. (2002) determine a visual extinction $A_{\mathrm{V}}=2.5 \pm 0.8$ towards HH240A. Assuming a standard gas-to-dust ratio $N_{\mathrm{H}}=$ $1.9 \times 10^{21} \mathrm{~cm}^{-2} \mathrm{mag}^{-1} A_{\mathrm{V}}$ (Bohlin et al. 1978), and that the HH 240A bow lies at a distance of $300^{\prime \prime}\left(2 \times 10^{18} \mathrm{~cm}\right.$ at the distance of 460 parsecs) from the edge of the L1634 cloud (from DSS2 image), we estimate an average hydrogen density of $\sim 2.5 \times 10^{3} \mathrm{~cm}^{-3}$.

\subsection{Modelling HH240A as a C-type bow}

After considerable exploration, we derived the parameters listed in Table 2 for HH 240A. For clarity and to highlight the modelling technique, we present here a discussion of the role of each of the parameters and the extent to which they influence the appearance of the bow.

We find a bow shape given by $s=2.35$ and scale $L_{\text {bow }}=$ $1.7 \times 10^{16} \mathrm{~cm}$ reproduces the overall wide angle and dimensionality of the bow. In the figures, we represent $L_{\text {bow }}$ by 9 pixels. This implies 1 pixel is 0 ". 28 . 
Table 3. Observed and predicted bow shock luminosities and $(2,1) \mathrm{S}(1) /(1,0) \mathrm{S}(1)$ flux ratios for $\mathrm{HH} 240 \mathrm{~A}$ and $\mathrm{HH} 240 \mathrm{C}$. Luminosities are in units of $L_{\odot}$. A source distance of $460 \mathrm{pc}$ is assumed. $K$-band extinctions of 0.28 and $1.0 \mathrm{mag}$ are applied to $\mathrm{HH} 240 \mathrm{~A}$ and $\mathrm{HH} 240 \mathrm{C}$ to determine the dereddened luminosities.

\begin{tabular}{lllll}
\hline \hline Line & Observed & Dereddened & $\begin{array}{l}\text { C-type } \\
\text { model }\end{array}$ & $\begin{array}{l}\text { J-type } \\
\text { model }\end{array}$ \\
\hline $\mathrm{HH} 240 \mathrm{~A}$ & & & & \\
\hline $\mathrm{H}_{2}(1,0) \mathrm{S}(1)$ & $2.6 \times 10^{-3}$ & $3.4 \times 10^{-3}$ & $4.4 \times 10^{-3}$ & $2.4 \times 10^{-3}$ \\
$\mathrm{H}_{2}(2,1) \mathrm{S}(1)$ & $2.8 \times 10^{-4}$ & $3.6 \times 10^{-4}$ & $5.5 \times 10^{-4}$ & $4.0 \times 10^{-4}$ \\
$2 / 1$ ratio & 0.11 & 0.11 & 0.12 & 0.17 \\
\hline $\mathrm{HH} 240 \mathrm{C}$ & & & & \\
\hline $\mathrm{H}_{2}(1,0) \mathrm{S}(1)$ & $1.9 \times 10^{-3}$ & $4.7 \times 10^{-3}$ & $7.7 \times 10^{-3}$ & $4.0 \times 10^{-3}$ \\
$\mathrm{H}_{2}(2,1) \mathrm{S}(1)$ & $2.8 \times 10^{-4}$ & $6.9 \times 10^{-4}$ & $1.0 \times 10^{-3}$ & $6.1 \times 10^{-3}$ \\
$2 / 1$ ratio & 0.15 & 0.15 & 0.13 & 0.15 \\
\hline
\end{tabular}

1. The low $\mathrm{H}_{2}(1,0) \mathrm{S}(1)$ luminosity of $2.6 \times 10^{-3} L_{\odot}$ limits the hydrogen density and bow velocity. A $K$-band extinction of 0.28 mag raises the intrinsic $(1,0) \mathrm{S}(1)$ luminosity to $3.4 \times 10^{-3} L_{\odot}$. We find that a low density of $n=2.5 \times 10^{3} \mathrm{~cm}^{-3}$ and a velocity of $60 \mathrm{~km} \mathrm{~s}^{-1}$ result in a sufficiently low model luminosity. Increasing the density to $n=4.0 \times 10^{3} \mathrm{~cm}^{-3}$ has the effect of doubling the luminosity. The total cooling calculated in the chosen bow model is $0.0044 L_{\odot}$ in the $(1,0) \mathrm{S}(1), 0.136 L_{\odot}$ in $\mathrm{H}_{2}$ (all rotational and vibrational) and $0.17 L_{\odot}$ total line cooling. Thus, as expected, $\mathrm{H}_{2}$ cooling dominates in low density bow shocks.

2. The molecular fraction is constrained to be $n\left(\mathrm{H}_{2}\right) /\left(n(\mathrm{H})+2 n\left(\mathrm{H}_{2}\right)\right)=n\left(\mathrm{H}_{2}\right) / n \sim 0.2 \pm 0.1$, in order to keep the luminosity low and to modify the vibrational excitation. Table 3 contains a detailed comparison of the observed and model characteristics. We assume in these initial models that the magnetic field direction is parallel to the direction of motion of the bow.

3. Emission nearer to the bow apex is characterised by higher molecular excitation since the shock strength is determined by the component of the bow speed transverse to the bow surface. The flux ratio between the $\mathrm{H}_{2}(2,1) \mathrm{S}(1)$ and $\mathrm{H}_{2}(1,0) \mathrm{S}(1)$ vibrational-rotational transition lines measures the vibrational excitation. According to the detailed physics and chemistry of C-type shocks, (Draine 1980; Draine et al. 1983), the ion-fraction and magnetic field strength determine the shock velocity at which molecular hydrogen dissociates. There will thus be a dissociated cap if $v_{\text {bow }}$ is greater than this critical breakdown velocity. We find that an Alfvén speed of $5 \mathrm{~km} \mathrm{~s}^{-1}$ and an ion-fraction of $1 \times 10^{-5}$ correctly reproduces the observed flux ratio and the position of dissociation, forward of which no $\mathrm{H}_{2}$ emission is observed. The shock thickness, inversely proportional to the ion fraction, is also then reproduced. In addition, we find that the high magnetic field is necessary to hold down the length of the $\mathrm{H}_{2}$ bow wings.

The cushioning effects of the magnetic field are illustrated in Fig. 7. A strong magnetic field results in a decrease of the
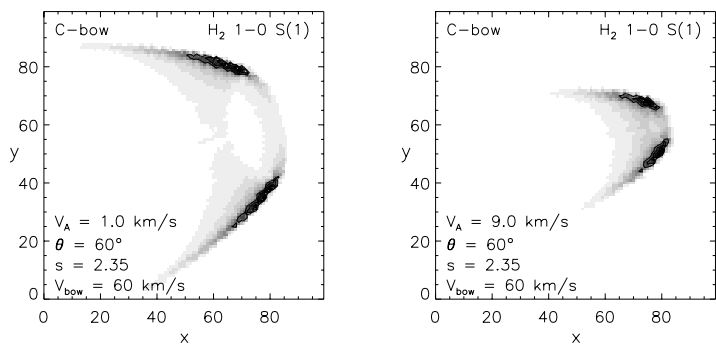

Fig. 7. C-bow models for HH 240A. The magnetic field strength (related through the Alfvén speed) determines the observed structure to a large extent. Other parameters are fixed to those in Table 2.
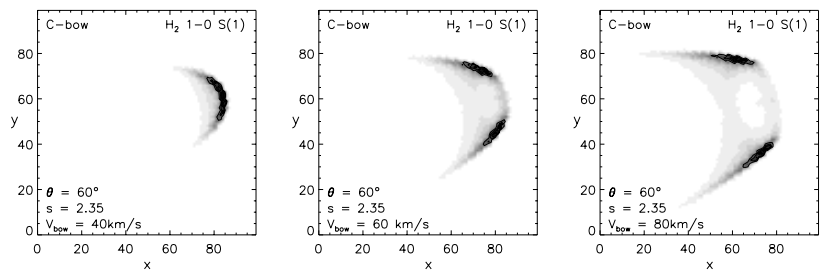

Fig. 8. C-bow models for HH 240A. The dependence of $(1,0) \mathrm{S}(1)$ emission on the bow shock velocity is shown here. The bow speed controls the compactness of the bow. Adopting a source distance of $460 \mathrm{pc}$ gives a pixel scale of 1 pixel $=0$ ' 28 . Other parameters are fixed to those in Table 2.

streaming velocity between the ions and the neutrals which allows the molecules to survive a stronger shock. In this case emission is seen closer to the front of the bow and it is inhibited in the wings. The cooling length increases with a higher magnetic field causing the emission to be located some distance back from the shock front, drastically altering the appearance of the bow. Lowering the magnetic field has the opposite effect.

4. The bow velocity is constrained by the observed flux distribution. Figure 8 shows that a bow velocity of $40 \mathrm{~km} \mathrm{~s}^{-1}$ results in a region of compact emission beginning at the bow apex. There is no dissociated cap because the dissociation speed is not exceeded. In contrast, a bow velocity of $80 \mathrm{~km} \mathrm{~s}^{-1}$ generates a bow with little emission from the front; most of the emission is contained in the extended wings. Between these extremes, a bow velocity of $60 \mathrm{~km} \mathrm{~s}^{-1}$ closely resembles the observed bow morphology.

5. The bow appearance depends on the direction of motion relative to the line of sight, as demonstrated in Fig. 9. An angle of $40^{\circ}$ results in a gaping hole behind a strong leading edge. In contrast, when the angle is $80^{\circ}$ no dip is present at all and the bow becomes over extended along the direction of motion in comparison to the observed HH 240A bow.

6. The bow structure is also very sensitive to the shape parameter $s$. Figure 10 explores this variable and we can see that a bow characterised by $s=2.65$ is too broad. When $s$ is lowered to 2.05 , the shape becomes too sharp. In this manner, the value of $s=2.35$ has been chosen to fit the appearance of the bow. The shape also influences the integrated $\mathrm{H}_{2}(2,1) \mathrm{S}(1)$ to $\mathrm{H}_{2}(1,0) \mathrm{S}(1)$ line flux ratio. The ratio increases from $0.107(s=2.05), 0.124(s=2.35)$ to 

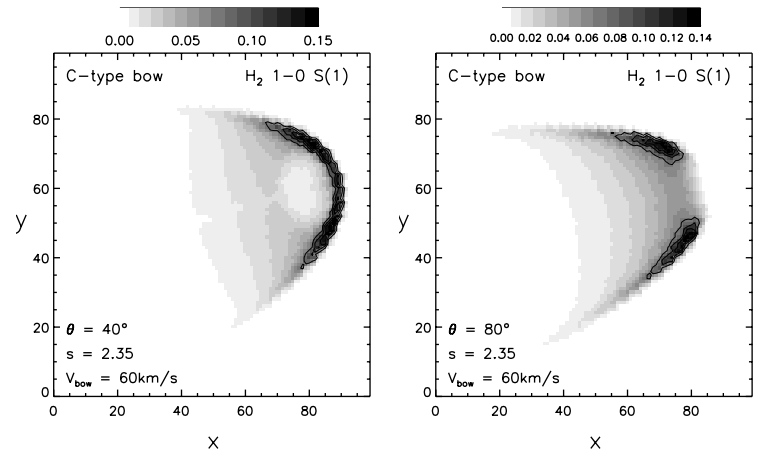

Fig. 9. C-bow models for HH 240A. Differences in the orientation between the bow shock motion and the line of sight drastically alter the appearance of the bow to the observer. Here we show C-type bow models with orientations of $80^{\circ}$ (right panel) and $40^{\circ}$ (left panel) to the line of sight. We find that for HH 240A an angle of $60 \pm 10$ degrees satisfactorily reproduces the observed image characteristics. Other parameters are fixed to those in Table 2.
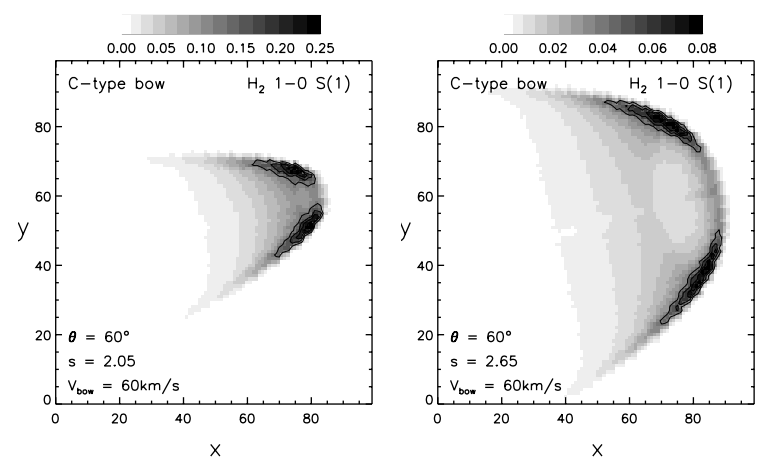

Fig. 10. The effect of altering the shape parameter $s$ is illustrated by these simulated $(1,0) \mathrm{S}(1)$ images of C-type bows with $s=2.05$ (left panel) and $s=2.65$ (right panel). Other parameters are fixed to those in Table 2.

$0.135(s=2.65)$. These values are in the observed range of $0.11 \pm 0.02$ (Table 3 ).

7. Axial symmetry in the model bow structure is broken through the magnetic field direction, as shown in Fig. 11. We have tried various angles $\mu$ and $\phi$ to better match the observations. A positive angle $\mu$ tends to increase the peak emission from the lower flank of the bow. The magnetic field provides greater cushioning along this edge and the symmetry is broken transverse to the line of sight. We find an angle $\mu=30^{\circ}$ and a somewhat higher bow speed of $v_{\text {bow }}=70 \mathrm{~km} \mathrm{~s}^{-1}$ provides an excellent fit. In the symmetric case, the vertical slit profile of the $(2,1) \mathrm{S}(1)$ emission is relatively stronger on the northern bow wing. This might be expected since the slit cuts through the bow nearer to the apex where the shock reaches higher temperatures. It is, however, contrary to the observed emission profiles shown in Fig. 2. Taking an oblique magnetic field, the excitation along the flanks is no longer symmetric. The southern peak may then be stronger in the $(2,1) \mathrm{S}(1)$ line, as indeed found and displayed in Fig. 11.

The theoretical rate at which a bow of speed $v_{\text {bow }}$ converts the driving energy into heat, $P$, is proportional to the bow area

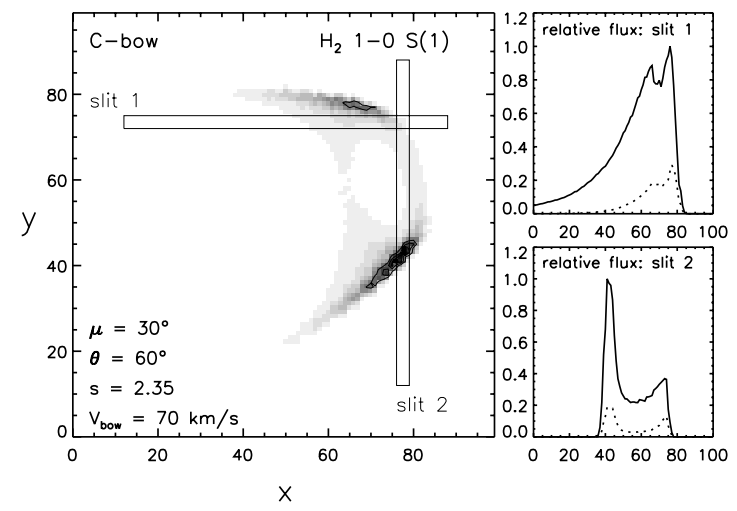

Fig. 11. A magnetic field oblique to the bow axis results in image asymmetry for C-type bows. Combined with a slightly higher bow speed, we are able to reproduce the observed structure. Note that we have adjusted the greyscale levels here to display less diffuse structure: the flux profiles demonstrate quantitatively the relative intensities. Other parameters are fixed to those in Table 2.

facing the oncoming flow, the energy flux and a nondimensional factor related to the aerodynamical drag. We combine these into the form $P=\zeta \rho v_{\text {bow }}^{3} L_{\text {bow }}^{2} / 2$ or

$$
\begin{aligned}
P= & 0.15 \zeta\left(\frac{n_{\mathrm{c}}}{2.5 \times 10^{3} \mathrm{~cm}^{-3}}\right)\left(\frac{v_{\text {bow }}}{60 \mathrm{~km} \mathrm{~s}^{-1}}\right)^{3} \\
& \times\left(\frac{L_{\text {bow }}}{1.7 \times 10^{16} \mathrm{~cm}}\right)^{2} L_{\odot},
\end{aligned}
$$

where $\zeta$ is of order unity, $n_{\mathrm{c}}$ is the density of hydrogen nucleons (and the mass density is $\rho=2.32 \times 10^{-24} n_{\mathrm{c}}$, including $10 \%$ helium atoms). Therefore, the derived numbers are consistent with expectations.

The mass outflow rate necessary to drive the bow can also be estimated. We use a somewhat larger driving jet speed, to obtain a twin-jet mass outflow rate of

$$
\begin{aligned}
\dot{M}_{\text {out }}= & 1.5 \times 10^{-6}\left(\frac{n_{\mathrm{c}}}{2.5 \times 10^{3} \mathrm{~cm}^{-3}}\right)\left(\frac{v_{\text {jet }}}{100 \mathrm{~km} \mathrm{~s}^{-1}}\right) \\
& \times\left(\frac{L_{\text {bow }}}{1.7 \times 10^{16} \mathrm{~cm}}\right)^{2} M_{\odot} \mathrm{yr}^{-1}
\end{aligned}
$$

This can be compared to the model-dependent infall rate derived by Beltrán et al. (2002) of $\dot{M}_{\text {in }}=2.6-8.0 \times 10^{-5} M_{\odot} \mathrm{yr}^{-1}$ which implies that well under $10 \%$ of the inflowing mass is ejected.

\subsection{Modelling the $\mathrm{HH} 240 \mathrm{C}$ image and power}

Although part of the same outflow, $\mathrm{HH} 240 \mathrm{C}$ has obvious differences to HH240A. No dissociated cap is apparent as the $(1,0) \mathrm{S}(1)$ emission appears to originate from close to the front of the bow. As a result, the model for this object employs a smaller and more focused bow geometry propagating through the cloud material with a lower velocity $\left(50 \mathrm{~km} \mathrm{~s}^{-1}\right)$ than found for $\mathrm{HH} 240 \mathrm{~A}$. We have again explored wide ranges of parameter space in determining the model shown in Fig. 12 and described in Table 2. 


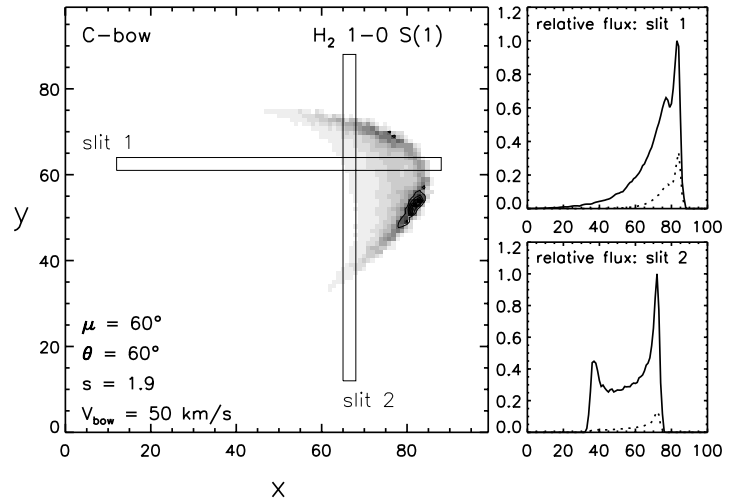

Fig. 12. A C-bow model for HH 240C. The $(1,0)$ S(1) emission image and flux profiles for the $(1,0) \mathrm{S}(1)$ (full line) and $(2,1) \mathrm{S}(1)$ (dotted line). Adopting a source distance of $460 \mathrm{pc}$ gives a pixel scale of 1 pixel $=0.221$. Other parameters are fixed to those in Table 2 .

HH 240C has a $(1,0) \mathrm{S}(1)$ luminosity comparable to HH 240A despite the smaller scale and speed. We therefore deduce that the bow is moving into a denser region where the hydrogen density is $7.0 \times 10^{3} \mathrm{~cm}^{-3}$. Although surprising, this is actually supported by the fact that a higher extinction is necessary for $\mathrm{HH} 240 \mathrm{C}$ in order to explain the populations in the $\mathrm{H}_{2}$ ro-vibrational energy levels (see Sect. 6).

In order to account for the asymmetry in the observed bow, we have introduced a magnetic field which is at an angle relative to the direction of motion of the bow (Smith 1991; Chernoff 1987). Referring to Fig. 6, we kept $\phi$ at zero and set $\mu$ equal to $60^{\circ}$. Therefore, the transverse components of the bow velocity range from being perpendicular to being parallel to the field lines as we move along the bow surface: the emission from one side of the bow front is enhanced and emission on the opposite side is depleted.

A value of $s=1.9$ was found in order to give the bow a more focused appearance. This, however, has the effect of reducing the excitation ratio as the wings become extended and emission from the cooler regions of the bow contribute relatively more. In order to counteract this effect, the fraction of ions present in the pre-shock is raised to $5.0 \times 10^{-5}$. This has the effect of increasing the excitation ratio to 0.133 , smaller than the measured value of 0.15 .

\subsection{Modelling with J-type shocks}

We have attempted to model HH 240A with a J-type bow shock in which the cap is dissociative and the flanks non-dissociative. Speeds exceeding $\sim 50 \mathrm{~km} \mathrm{~s}^{-1}$ generate long tails of strong $(1,0) \mathrm{S}(1)$ emission. We find speeds of $\sim 40 \mathrm{~km} \mathrm{~s}^{-1}$ produce structure reminiscent of that observed but only when combined with a relatively strong magnetic field. An oblique field angle also improves the fit to the flux profiles.

In order to obtain a reasonable luminosity, we model with a higher density. Although we have found it difficult to simultaneously produce an excitation as low as observed and a sufficiently high luminosity, our best fit shown in Fig. 13 does approach observed values. The model yields a $(1,0) \mathrm{S}(1)$ luminosity of $2.4 \times 10^{-3} L_{\odot}$ and a $(2,1) \mathrm{S}(1) /(1,0) \mathrm{S}(1)$ luminosity
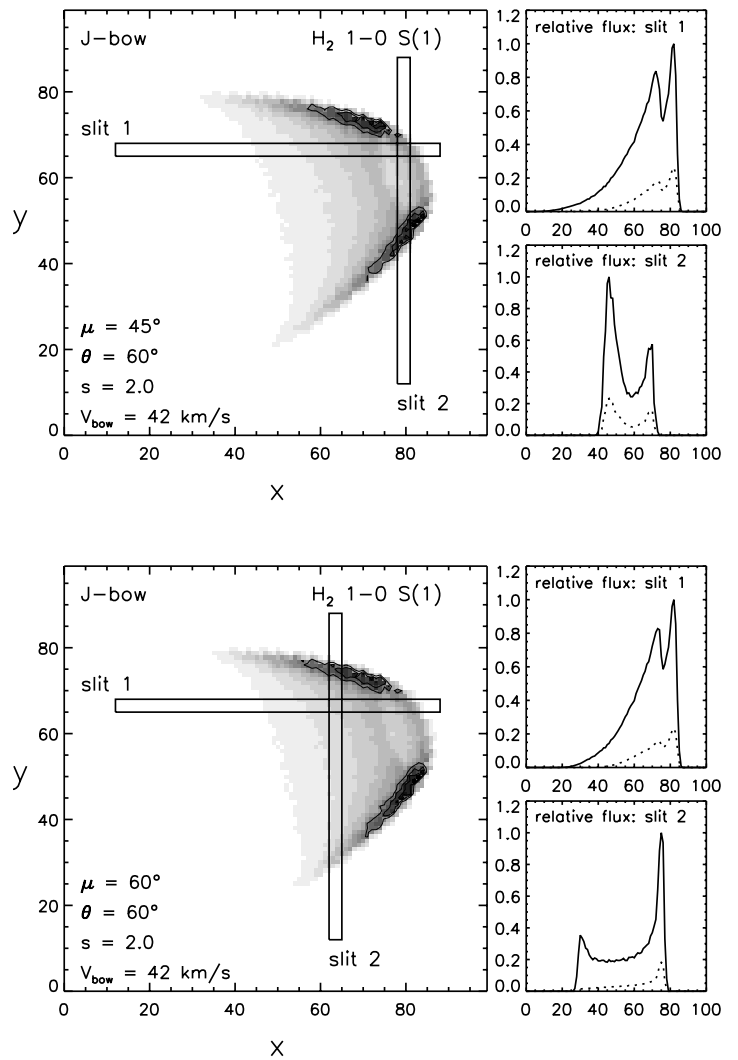

Fig. 13. The best fit J-type bow model for the $(1,0) \mathrm{S}(1)$ emission from HH240A (top) and HH240C (lower). The pixel scales are 1 pixel $=0$ ".28 (top) and 0".21 (lower). The full parameter set is listed in Table 4 . The model yields a $(1,0) \mathrm{S}(1)$ luminosity of $2.4 \times 10^{-3} L_{\odot}$ and a $(2,1) \mathrm{S}(1) /(1,0) \mathrm{S}(1)$ luminosity ratio of 0.17 for $\mathrm{HH} 240 \mathrm{~A}$ and $4 \times 10^{-3} L_{\odot}$ and a $(2,1) \mathrm{S}(1) /(1,0) \mathrm{S}(1)$ luminosity ratio of 0.15 for HH $240 \mathrm{C}$.

Table 4. Model parameters derived to fit the bows with J-type shocks.

\begin{tabular}{lll}
\hline \hline Parameter & HH 240A & HH 240C \\
\hline Size, $L_{\text {bow }}$ & $1.7 \times 10^{16} \mathrm{~cm}$ & $1.3 \times 10^{16} \mathrm{~cm}$ \\
H density, $n$ & $5.0 \times 10^{3} \mathrm{~cm}^{-3}$ & $2.0 \times 10^{4} \mathrm{~cm}^{-3}$ \\
Molecular fraction & 0.2 & 0.3 \\
Alfvén speed & $5 \mathrm{~km} \mathrm{~s}^{-1}$ & $6 \mathrm{~km} \mathrm{~s}^{-1}$ \\
Magnetic field & $191 \mu \mathrm{G}$ & $458 \mu \mathrm{G}$ \\
Ion fraction & $>10^{-4}$ & $>10^{-4}$ \\
Bow velocity & $42 \mathrm{~km} \mathrm{~s}^{-1}$ & $42 \mathrm{~km} \mathrm{~s}^{-1}$ \\
Angle to l.o.s. & $60^{\circ}$ & $60^{\circ}$ \\
$s$ parameter & 2.0 & 2.0 \\
Field angle, $\mu$ & $45^{\circ}$ & $60^{\circ}$ \\
\hline
\end{tabular}

ratio of 0.17 . Parameters for this model are listed in Table 4. In general, J-type bows show high excitation unless geometrically long tails (low values of $s$ ) are taken. This, however, generates long tails of $(1,0) \mathrm{S}(1)$ emission.

The general structure of $\mathrm{HH} 240 \mathrm{C}$ can be modelled with a J-type bow, as shown in the lower panels of Fig. 13. For $\mathrm{HH} 240 \mathrm{C}$ we again require a high magnetic field to inhibit the formation of a long tail. A high density is also essential to produce the luminosity from such a small J-type bow. The density is 3 times higher than required for the C-type bow. The 


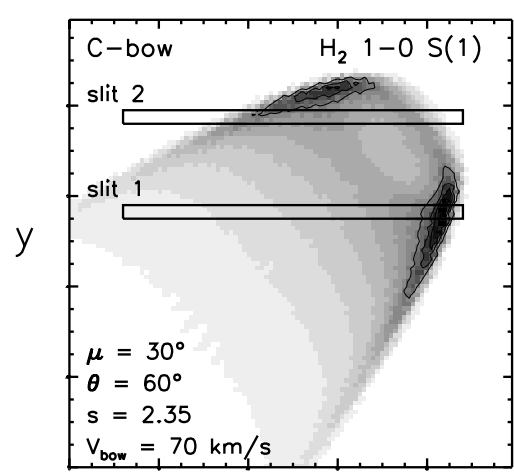

$x$
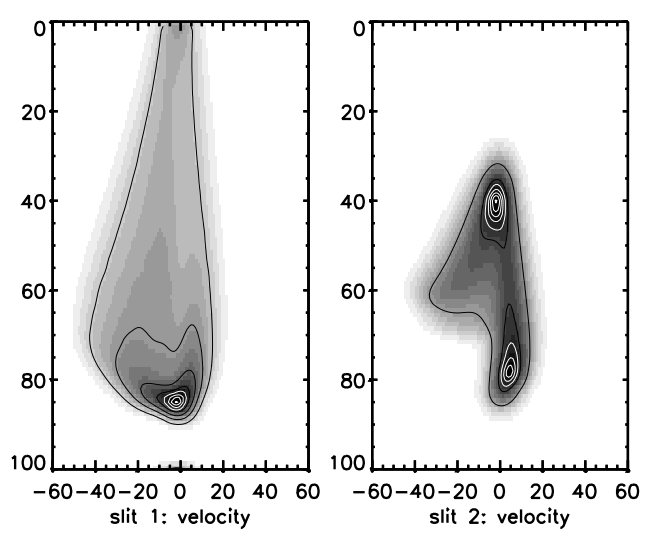

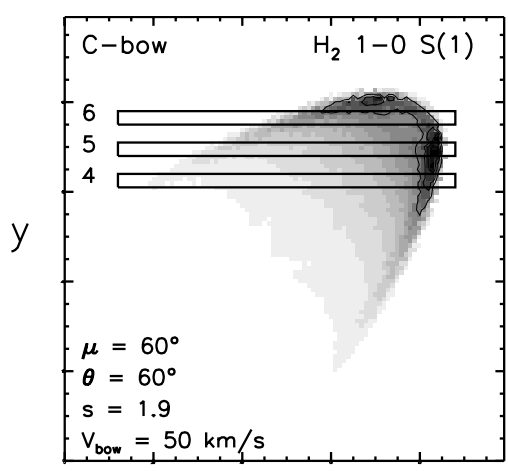

$\mathrm{X}$
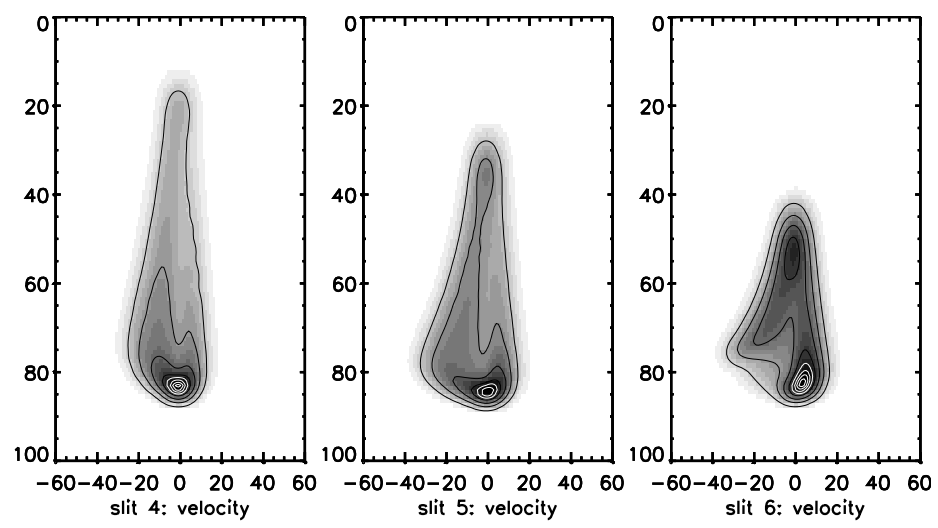

Fig. 14. Position-velocity diagrams for our best-fit C-type bow models for HH 240A (top) and HH 240C (bottom). The modelled line is the $\mathrm{H}_{2}(1,0) \mathrm{S}(1)$. Slit positions are indicated and labelled to correspond to the observations. The bows and slits have been rotated for display purposes. The flow axis is $-75^{\circ}$ and the slit axis is $+63^{\circ}$. We have applied a Gaussian standard deviation of $4.3 \mathrm{~km} \mathrm{~s}^{-1}$ to match the instrumental accuracy.

model yields a $(1,0) \mathrm{S}(1)$ luminosity of $4.0 \times 10^{-3} L_{\odot}$ and a $(2,1) \mathrm{S}(1) /(1,0) \mathrm{S}(1)$ luminosity ratio of 0.15 . A lower velocity than for HH 240A is not necessary, and a field at a large angle to the bow direction is favoured (see Table 4).

Our main conclusions are that HH 240A corresponds exceedingly well to a C-type bow shock model in both image and excitation. HH240C corresponds closer to a J-type bow in excitation but the structure can be modelled by either shock physics. Quite high and oblique magnetic fields are predicted. As we will find below, the magnetic field inferred from the $\mathrm{H}_{2}$ modelling is contained within processed outflowing gas rather than ambient molecular cloud gas.

\section{Modelling the velocity distribution}

\subsection{C-type bows}

Independent of the imaging, the derived bow shock models can be tested against the position-velocity $(\mathrm{P}-\mathrm{V})$ data in the $\mathrm{H}_{2}(1,0) \mathrm{S}(1)$ emission line. We have generated P-V diagrams for our best-fit C-type models. We have re-orientated the bow axis and the slits in Fig. 14 for display purposes, maintaining the angle of $42^{\circ}$ between the two directions. Model slit numbers correspond to the observed slits shown in Fig. 4.

For the HH240A model (upper panels), the simulated $\mathrm{P}-\mathrm{V}$ structure resembles the observed $\mathrm{P}-\mathrm{V}$ structure. Along
Slit 1 , we find a broad velocity width with a strong leading peak at low velocity although the observed weak extension ahead of the bow at high blue-shifted speeds is not reproduced here.

A narrowing tail along the slits approaches zero velocity. Note that the observed tail also approaches zero radial velocity, although the background cloud has a radial speed of $+8 \mathrm{~km} \mathrm{~s}^{-1}$ (De Vries et al. 2002). In other words, the bow moves within gas which is already in motion with a radial component of $-8 \mathrm{~km} \mathrm{~s}^{-1}$. Given the derived orientation, the pre-shock outflow speed is estimated to be $\sim 16 \mathrm{~km} \mathrm{~s}^{-1}$.

Simulated Slit 2 possesses a second strong peak located towards the back. Towards the apex, the rear and near bow wings combine to produce an increasing width with a very sharp edge at zero velocity. These features are also observed. However, the leading peak is slightly redshifted in the simulated bow (since the projected leading edge is on the rear side of the bow). This structure is not apparent in the observed bow which has a quite broad-width leading edge.

For the HH 240C model (lower panels), some of the structure is reproduced. For Slit 4, the simulated diagram has a strong peak followed by two tails separated in velocity by about $15 \mathrm{~km} \mathrm{~s}^{-1}$ and a very extended tail. This has an overall similarity to the observed structure but the full velocity width of the observed line, $\sim 80 \mathrm{~km} \mathrm{~s}^{-1}$, is much greater. 

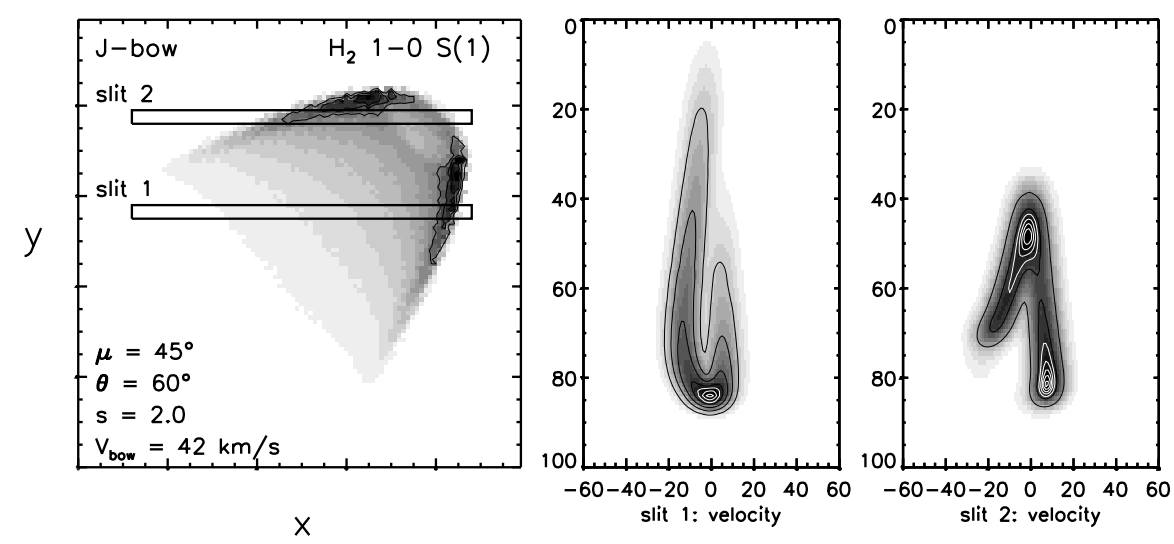

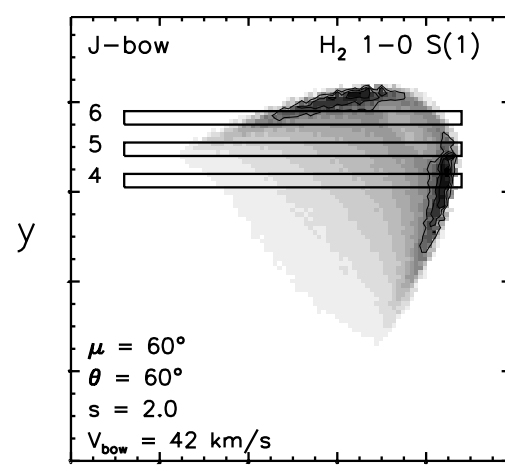

$X$
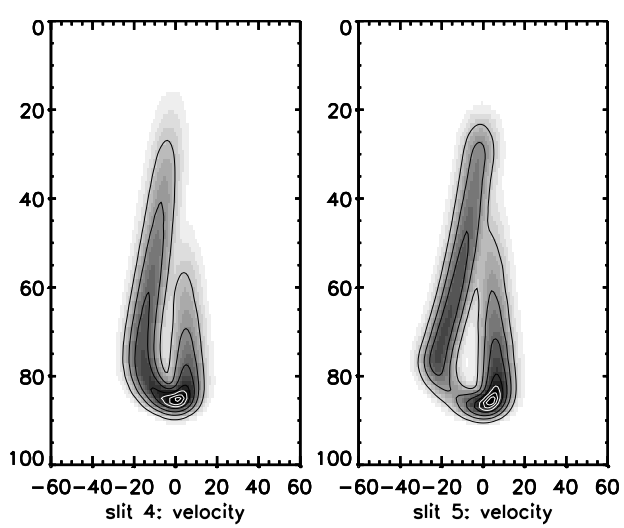

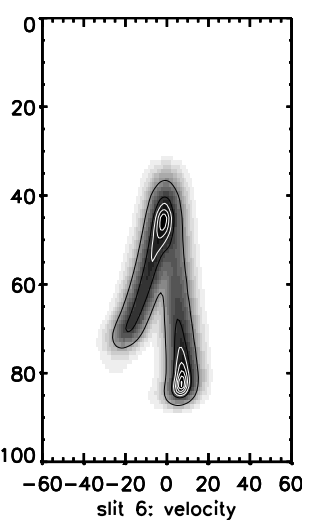

slit 6: velocity

Fig. 15. Position-velocity diagrams for our best-fit J-type bow models for HH 240A (top) and HH 240C (bottom). The modelled line is the $(1,0) \mathrm{S}(1) \mathrm{H}_{2}$. Slit positions are indicated and labelled to correspond closely to the observations. Thus, the bows and slits have been rotated for display purposes. The bow flow axis is at a position angle of $-75^{\circ}$ and the slit axis is $+63^{\circ}$. We have applied a Gaussian standard deviation of $4.3 \mathrm{~km} \mathrm{~s}^{-1}$ to match the instrumental accuracy.

For Slit 5, the strong low-velocity peak and the twin velocity-separated wings are similar to the observed features (although the blue wing possesses a distinct peak). For Slit 6, two peaks are predicted, each with two linear extensions. The two linear extensions are indeed observed but somewhat more overlapping in position. Full widths of $\sim 50-60 \mathrm{~km} \mathrm{~s}^{-1}$ are predicted, which do match the observations.

In general, much of the observed $\mathrm{P}-\mathrm{V}$ structure is reproduced in the simulated bows. However, it is clear that a clumpy medium, along with dynamical or thermal instability (see, for example, Hester et al. 1998), is leading to fine structure and line broadening in the bows.

\subsection{J-type bows}

Corresponding P-V diagrams for the best-fit J-type models are presented in Fig. 15. For HH 240A, J-type bow models predict very narrow $\mathrm{H}_{2}$ lines. The near and far wings of the bow are well separated in velocity space. This is as expected since the $(1,0) \mathrm{S}(1)$ emission is not generated immediately behind the J-shock jump where the gas is relatively hot. Instead, the emission arises from material which has been deflected, decelerated and re-cooled down to under $\sim 4000 \mathrm{~K}$. The resulting structure does not resemble $\mathrm{HH} 240 \mathrm{~A}$.

For HH 240C, the two distinct velocity wings along Slits 4 and Slits 5 are hinted at in the observations. Especially noteworthy is the convergence of the two wings in Slit 5 of Fig. 15 which has a corresponding structure in the observed Slit 5. Slit 6 possesses two very distinct linear features, again resembling the observed filamentary structure.

The molecular breakdown speed, $v_{\mathrm{d}}$, is defined as the maximum speed of a planar shock in which molecules survive in significant numbers. The full width of the lines from bow shocks have a theoretical upper extent of twice the breakdown speed (Smith \& Brand 1990). To reach this maximum line width, however, the bow must be moving in the plane of the sky as well as at a speed far in excess of the breakdown speed (otherwise, the shocked gas is accelerated more towards the bow axis than transversely). At an angle of $60^{\circ}$ to the line of sight the maximum line width is approximately $2 v_{\mathrm{d}} \sin 60^{\circ}$ (Smith \& Brand 1990).

For J-type bows, the predicted line widths can be close to the maximum since the emission from $\mathrm{H}_{2}(1,0) \mathrm{S}(1)$ arises from the deflected and cooled gas. Given also the strong magnetic field and the quite low density, we find $v_{\mathrm{d}} \sim$ $35 \mathrm{~km} \mathrm{~s}^{-1}$ (Smith 1994). Therefore, full line widths can approach $55 \mathrm{~km} \mathrm{~s}^{-1}$ for the $\mathrm{HH} 240$ flow. This value is consistent with that found for the simulated bows and also with some of the observations but not the observed widths within HH240A.

To summarise these results, HH240A can be interpreted as a C-type bow, according to structure, excitation and velocity distribution. On the other hand, HH 240C possesses elements of both $\mathrm{J}$ and C-type shocks, neither of which on 
Table 5. Predicted infrared line fluxes from the C-type bow shock model for HH240A and the J-type bow model for HH 240C, as defined in Tables 2 and 4, respectively.

\begin{tabular}{lrll}
\hline \hline Line & $\begin{array}{rll}\text { Wavelength } \\
\mu \mathrm{m}\end{array}$ & $\begin{array}{l}\text { HH 240A } \\
L_{\odot}\end{array}$ & $\begin{array}{l}\text { HH 240C } \\
L_{\odot}\end{array}$ \\
\hline $\mathrm{H}_{2}$ 0-0 S(5) & 6.9 & $2.1 \times 10^{-2}$ & $4.7 \times 10^{-3}$ \\
$\mathrm{H}_{2}$ 0-0 S $(1)$ & 17.0 & $2.1 \times 10^{-3}$ & $1.1 \times 10^{-3}$ \\
$\mathrm{O} \mathrm{I}$ & 63 & $6.9 \times 10^{-3}$ & $3.2 \times 10^{-2}$ \\
$\mathrm{CO} J=30-29$ & 87 & $5.0 \times 10^{-5}$ & $2.8 \times 10^{-4}$ \\
$\mathrm{CO} J=20-19$ & 130 & $7.1 \times 10^{-4}$ & $3.0 \times 10^{-3}$ \\
$\mathrm{CO} J=10-9$ & 260 & $5.1 \times 10^{-3}$ & $1.0 \times 10^{-3}$ \\
$\mathrm{C} \mathrm{I}$ & 372 & $1.1 \times 10^{-3}$ & $9.3 \times 10^{-4}$ \\
\hline
\end{tabular}

its own provides a full interpretation. Predicted infrared luminosities from these bow models are listed in Table 5. The major differences result from the higher density in the J-type model along with a higher compression before emission lines from cooler gas (often with long wavelength) are produced. Rovibrational $\mathrm{H}_{2}$ cooling dominates across the low-density C-type bow.

\subsection{The HH 241 system of shocks}

HH 241A possesses spatial structure similar to HH 240A with two strong $\mathrm{H}_{2}$ bow flanks. This bow appears on all 5 slits, Slits 8-12, appearing in Fig. 5 at locations 1-25. Speeds of $+50 \mathrm{~km} \mathrm{~s}^{-1}$ are detected along the bow axis (Slit 10). Positive radial speeds of $20-30 \mathrm{~km} \mathrm{~s}^{-1}$ are found in the wings. There are clear departures in the overall velocity from that found in $\mathrm{HH} 240$. Most significant is that there is very little emission from between 0 and $20 \mathrm{~km} \mathrm{~s}^{-1}$ on both sides of the outflow; what emission there is could occur from deflection by the flanks of the bows nearer to the plane of the sky.

$\mathrm{HH} 241 \mathrm{~B}$ possesses a higher $\mathrm{H}_{2}$ velocity dispersion at its western edge (i.e. positions 40-50 in Slits 9 and 10 of Fig. 5). This is the side facing the driving source and so we can interpret this as a reverse bow in the flow. The spatial structure provides some support although the wings do not appear to be well developed.

\section{Column density distributions}

\subsection{Extinction modelling}

A fully consistent model must account for the observed emission fluxes from various other molecular hydrogen lines. Many fluxes, originating from several vibrational levels, have been measured by Nisini et al. (2002). We have calculated here the $\mathrm{H}_{2}$ column densities in the upper energy levels $T_{\mathrm{j}}$ of the transitions required to produce these fluxes. The derived columns can be directly modelled in terms of an excitation temperature. The columns range over several orders of magnitude although individual error bars are under $20 \%$ for many lines. This is mainly because the gas temperature is in the range $2000-3000 \mathrm{~K}$ whereas upper energy levels range from $6000 \mathrm{~K}$ to above $20000 \mathrm{~K}$. Therefore, to make the error bars visible, we divide each column by the predicted column from molecular gas taken to be at a fixed $2000 \mathrm{~K}$, a method first employed by Brand et al. (1988). The resulting quantities are the Column Density Ratios (CDRs) for which the strong exponential dependence of the columns on the upper energy level has been removed. For modelling convenience, we express the CDRs relative to the CDR for the upper level, $v=1, J=3$ of the $(1,0) \mathrm{S}(1)$ line at $6956 \mathrm{~K}$.

The first factor to constrain with a CDR diagram is the extinction to the bow shocks. Extinction acts differentially, to mainly reduce the observed fluxes from shorter wavelengths. Ideally, we would employ lines originating from the same upper energy level but at different wavelengths for which the intrinsic flux ratio is a well-determined physical constant. However, the only pairs of lines with this property in the data set involve $(1,0)$ Q-branch lines with wavelengths $>2.4 \mu \mathrm{m}$. The fluxes of these lines are notoriously unreliable from ground-based observations.

We constrain extinction here by applying physical constraints, following the method described by Smith et al. (2003a). We show CDR diagrams for HH240C in Fig. 16. The data in the panels differ by the amount of imposed foreground extinction. For no extinction (top panel) the data possess a wide scatter transverse to the overall trend. We have then gradually increased the extinction until the tightest correlation was found, checking for consistency with the non-LTE conditions we expect at densities near $10^{4} \mathrm{~cm}^{-3}$. We thus found that HH $240 \mathrm{C}$ lies behind $1 \pm 0.2$ mag of $K$-band extinction (middle and lower panels). Adopting an extinction law of the form $\lambda^{-1.7}$, this is sufficient to modify the excitation we derive from our $(2,1) /(1,0)$ flux ratio by a small amount, decreasing the excitation by about $10 \%$.

A similar analysis for $\mathrm{HH} 240 \mathrm{~A}$ demonstrates that very little extinction is permitted (see Fig. 17). We estimate an extinction $\left(A_{K}\right)$ of $0.28 \pm 0.2$ from the CDR data. This value is consistent with the low extinction found by Nisini et al. (2002) from a near infrared [Fe II] line analysis. These [Fe II] lines were not detected in $\mathrm{HH} 240 \mathrm{C}$ so we cannot check for consistency. The non-detection is probably consistent with the low speed of HH 240C.

For $\mathrm{HH} 241 \mathrm{~A}$ and $\mathrm{HH} 241 \mathrm{~B}$, there is also sufficient data to constrain the extinction. We find $K$-band extinctions of $0.25 \pm 0.15(\mathrm{HH} 241 \mathrm{~A})$ and $0.3 \pm 0.1(\mathrm{HH} 241 \mathrm{~B})$. Therefore, high extinctions are excluded even though this side of the outflow is moving away from the observer. Nisini et al. (2002) derive a $K$-band extinction of $0.45 \pm 0.1$ from the [Fe II] line analysis of $\mathrm{HH} 241 \mathrm{~A}$, consistent with our $\mathrm{H}_{2}$ CDR range.

\subsection{Bow shock models}

We fit HH 240C with our best-fit C-type model in Fig. 16. Note that the three lines correspond to the first three vibrational levels, with the solid line modelling the $(1,0)$ (square) data points, the dotted line modelling the $(2,1)(\mathrm{X})$ data points and the dotdash line modelling the 3-2 (triangular) data points. The agreement is quite good although there is a general tendency to underpredict the higher level excitation. Note also the large scatter 

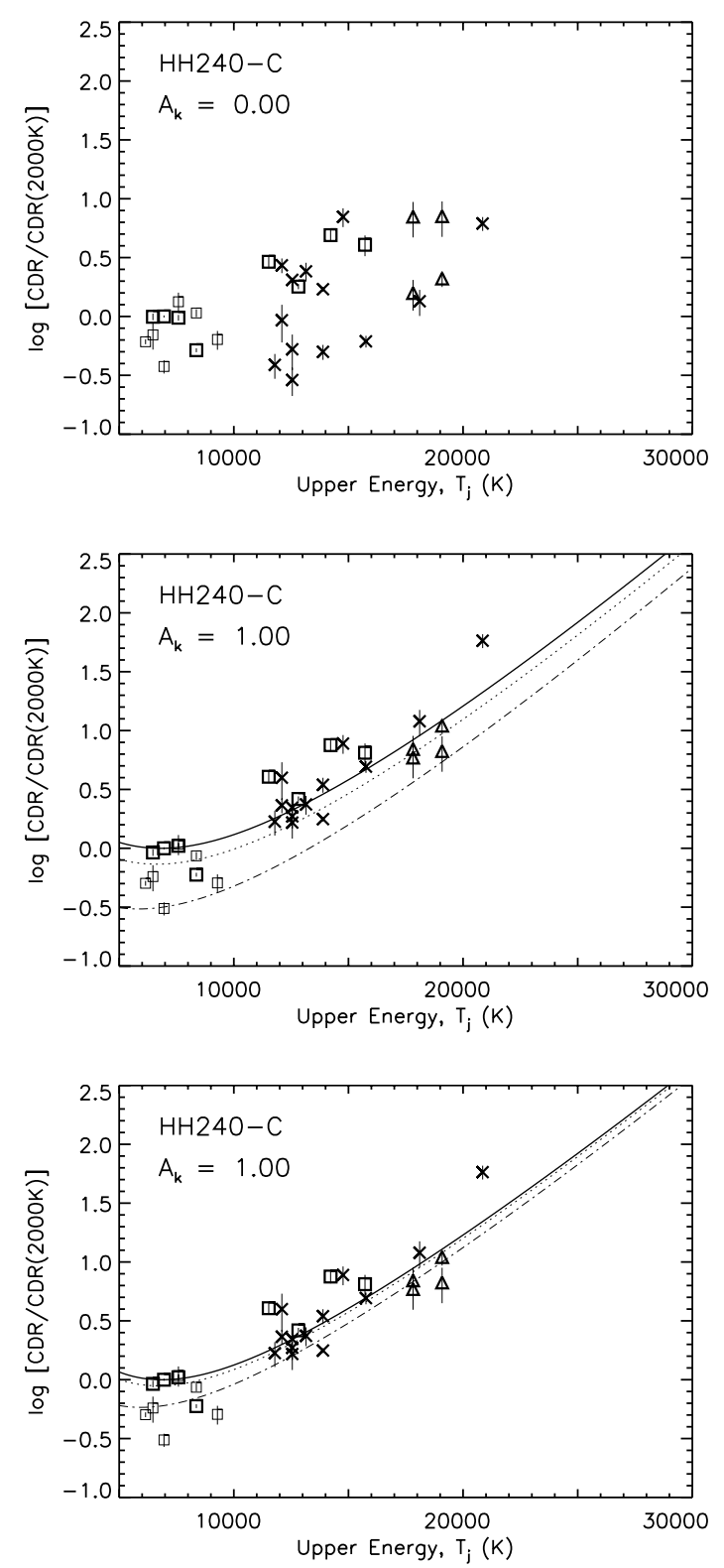

Fig. 16. Column Density Ratio diagrams for $\mathrm{HH} 240 \mathrm{C}$ evaluated from line fluxes presented by Nisini et al. (2002). The top panel presents the data assuming no extinction. The symbols represent $\mathrm{H}_{2}(1,0)$ (squares), $(2,1)$ and 2-0 (crosses), the 3-2 and 3-1 (triangular) data points. The faint squares represent $(1,0) \mathrm{Q}$ branch measurements. The middle panel demonstrates the much better intrinsic consistency when one magnitude of $K$-band extinction is applied. Superimposed is the C-type best fit model lines with three vibrational levels, solid (first), dotted (second) and dot-dashed (third) represented. The lower panel displays the corresponding best-fit J-type bow.

in the Q-branch data points, represented by faint square symbols, while the discrepant $(1,0) \mathrm{S}(3)$ line flux at $T_{\mathrm{j}}=8000 \mathrm{~K}$ is definitely measurement error, lying on the far opposite edge of the $K$-band window.

The J-type bow model is also displayed in the lower panel of Fig. 16. The major difference is that the vibrational levels are much closer to LTE. This is a consequence of the higher density as well as the nature of a J-type shock. Although the

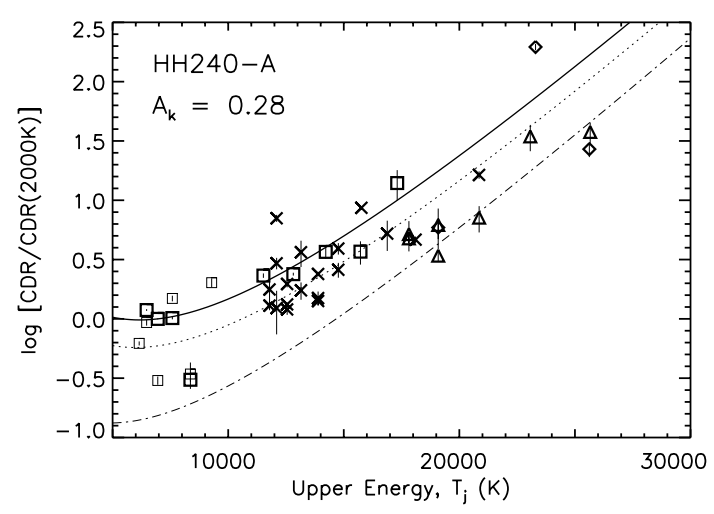

Fig. 17. Column Density Ratio diagram for HH 240 A evaluated from line fluxes presented by Nisini et al. (2002). Details are as described in Fig. 16. The model lines are taken from the best fit C-type bow model. The symbols represent $\mathrm{H}_{2}(1,0)$ (squares), $(2,1)$ and $2-0$ (crosses), the 3-2 and 3-1 (triangular) and 4-2 (diamond) data points.
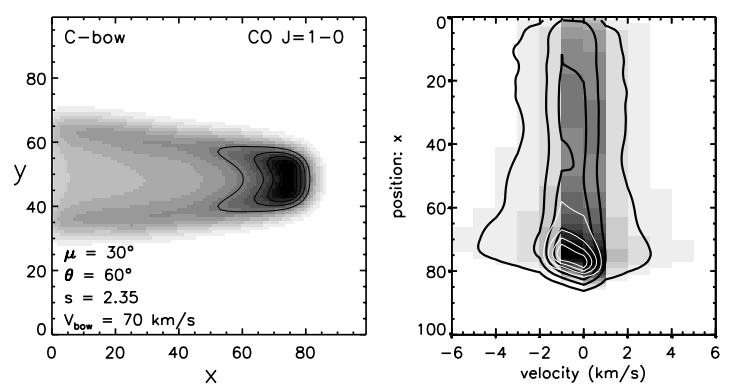

Fig. 18. $\operatorname{CO} J=(1,0)$ emission with pixel size of $11^{\prime \prime} 24$ from the best-fit bow model for HH 240A.

J-type model better fits the higher vibrational levels, neither model can be dismissed.

We fit HH 240A with our best-fit C-type model in Fig. 17. The C-type bow model provides an excellent fit to all three vibrational levels. This provides yet more support for this model for HH 240A. In contrast, we find that the J-type bow overpredicts emission from the 3-2 lines with the predicted columns being much closer to LTE (not displayed).

\section{CO structure}

CO imaging and spectroscopy in the $J=(1,0)$ line of the L1634 outflow have been presented by Lee et al. (2000). They demonstrated that cold gas in motion is associated with HH240A, where it curves around with the $\mathrm{H}_{2}$ shape.

For comparison, we display the predicted $\mathrm{CO}$ image and $\mathrm{P}-\mathrm{V}$ diagram in Fig. 18 employing the C-bow model for HH 240A discussed above (and applying the non-LTE approximation of McKee et al. 1982). Note the large image scale employed in which a long CO tail is exhibited. The two panels are comparable to the $\mathrm{CO}$ map and $\mathrm{P}-\mathrm{V}$ diagrams of Lee et al. (2000), where the large tailed bow was found associated with HH 240A. More blue emission, however, is observed to be associated with the southern wing, not predicted here.

The main observed features on the $\mathrm{CO} \mathrm{P}-\mathrm{V}$ diagram are also reproduced with the $\mathrm{C}$-shock model. The model bow, 
however, does not predict the observed strong red peak at $+1 \mathrm{~km} \mathrm{~s}^{-1}$ although a red extension feature is simulated.

Most remarkable are the radial speeds of the gas components near the HH 240A and HH 241 bow shocks. The molecular cloud radial speed is $+8 \mathrm{~km} \mathrm{~s}^{-1}$. One would expect that the $\mathrm{CO}$ gas would also show the flux peak near $\sim+8 \mathrm{~km} \mathrm{~s}^{-1}$, as indeed found. However, the $\mathrm{H}_{2}$ radial speeds associated with the HH 240 bows is almost exclusively $<0 \mathrm{~km} \mathrm{~s}^{-1}$ while that associated with the $\mathrm{HH} 241$ bows is almost exclusively $>+20 \mathrm{~km} \mathrm{~s}^{-1}$. According to our simulated P-V diagrams, the radial speed ahead of both $\mathrm{HH} 240 \mathrm{~A}$ and $\mathrm{HH} 240 \mathrm{C}$, as given by the tail emission as well as the modelling of the leading edge, should be the same for both the $\mathrm{H}_{2}$ and $\mathrm{CO}$, contrary to what is observed.

The resolution of this problem is probably that the accelerated $\mathrm{CO}$ gas and the excited $\mathrm{H}_{2}$ gas possess distinctly different origins. We require the existence of twin outflow channels which lie within the static background cloud. The mean outward speed in the two oppositely-directed channels is $\sim 20 \mathrm{~km} \mathrm{~s}^{-1}$, yielding radial speeds relative to the cloud of $\pm 10 \mathrm{~km} \mathrm{~s}^{-1}$. Bow shocks begin to propagate within these channels at typical speeds of $\pm 80-90 \mathrm{~km} \mathrm{~s}^{-1}$ relative to the cloud, yielding bow speeds $\pm 60-70 \mathrm{~km} \mathrm{~s}^{-1}$ relative to the channel. These bows produce the $\mathrm{H}_{2}$ emission. The CO, emission, however, is predominantly produced from the ambient cloud immediately surrounding the channels, set in motion by weak expanding shock fronts prompted by the outflow and the bow shock HH 240A, in particular.

No bow shock beyond HH240A is detectable in CO. One explanation would be that the $\mathrm{CO}$ is depleted from the cloud in this region. Although we derive a higher density, from both the $\mathrm{H}_{2}$ luminosity and the $\mathrm{H}_{2}$ column ratios, the density would appear insufficient to cause a high depletion. Our favoured explanation is that the dense gas is distributed within sheets and clumps occupying a small fraction of the volume. Since the $\mathrm{CO}$ emission traces all the gas set in motion, whereas the $\mathrm{H}_{2}$ emission traces just the presently shocked gas, the $\mathrm{CO}$ emission may not be detectable. The high density and magnetic field would be consistent with the HH 240C bow now entering one such clump. The clump has been formed by the passage of an earlier shock which has raised both the magnetic field and the Alfvén speed during the cooling and compression. Upon entering, the bow is transforming from J-type to C-type due to a large drop in ionisation fraction. The time scale for the transformation is estimated to be $L_{n} / v_{\mathrm{A}}$ where $L_{n}$ is the thickness of a C-shock (Smith \& Mac Low 1997). The thickness is of order $\chi 10^{15} \mathrm{~cm} / n$ where $\chi$ is the ion fraction. Taking values from Table 2 yields a timescale of $\sim 4 \times 10^{9} \mathrm{~s}$ which is of the same order as the bow travel time, $d / v_{\text {bow }} \sim 3 \times 10^{9} \mathrm{~s}$. Therefore, it is very likely that we would capture a shock undergoing the neutral transformation stage (see Smith \& Mac Low 1997).

This picture then implies that there is a cloud edge near HH 240A beyond which there is only fragmentary but compressed dense cloud material. This material may be the result of the sweeping effect of previous bow shock fronts.

The above discussion leads us to suggest that, while HH 240A is almost certainly a C-shock, HH 240C is a J-shock

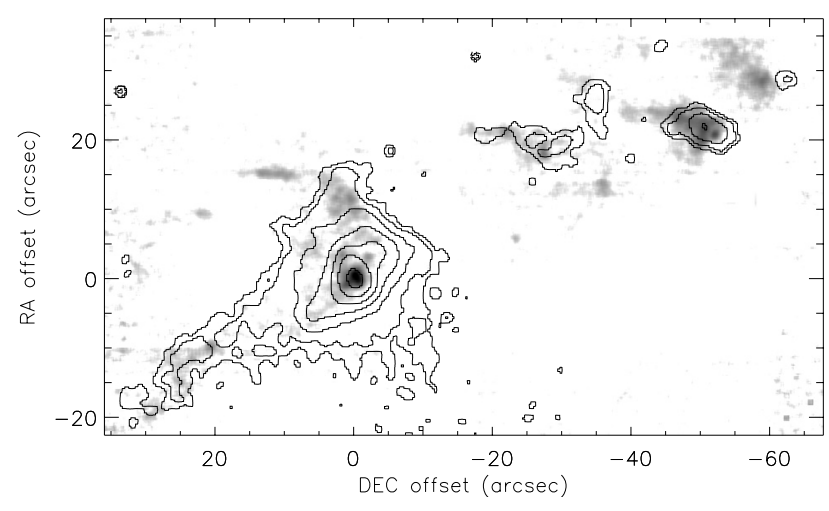

Fig. 19. An [S II] optical image of HH 240 kindly provided by Bohigas, as described in (Bohigas et al. 1993), roughly superimposed as a contour image over our $\mathrm{H}_{2}(1,0) \mathrm{S}(1)$ image. Contour levels increase logarithmically and represent $0.3,0.8,1.9,4.8,12,30$ and $76 \%$ of the maximum value. The alignment between the data sets was done manually. Nevertheless, the figure demonstrates that atomic and molecular emission is associated with each $\mathrm{HH}$ knot.

with a developing magnetic precursor. The contrasting shock physics explains the contrasting $\mathrm{H}_{2}$ vibrational excitation.

\section{Optical structure}

Atomic emission lines have been studied in the optical and the near-infrared. Measurements of [O III] lines imply high shock speeds near HH 240A. In contrast, [S II] lines suggest low speeds (Bohigas et al. 1993). The contrasting speeds could be reconciled by introducing a very high speed bow shock in which the $[\mathrm{SII}]$ is produced from the highly oblique bow wings. Bohigas et al. (1993) suggest a shock speed of $260 \mathrm{~km} \mathrm{~s}^{-1}$, based on a specific [O III] line ratio. This is, however, inconsistent with the proper motions and the location of the $\mathrm{H}_{2}$ emission just offset from the apex. This then suggests that a large fraction of the optical emission arises from the impact of a fast jet which drives the slow bow shock through the outflow. The structure and location of the optical emission is actually consistent with three components: a slender jet (seen in $\mathrm{H} \alpha$ ), a compact Mach disk (seen in [O I] and [N II]) located at HH 240A, and the bow shock (seen in [S II] and $\mathrm{H}_{2}$ ), all presented by Bohigas et al. (1993). The approximate locations of the [S II] and $\mathrm{H}_{2}$ are also illustrated in Fig. 19.

We note that the $[\mathrm{O} \mathrm{III}](4959+5007) / \mathrm{H} \beta$ flux ratio in $\mathrm{HH} 240 \mathrm{~A}$ is 0.3 . This is consistent with a planar shock of speed 90-100 $\mathrm{km} \mathrm{s}^{-1}$ according to Table 1 of Hartigan et al. (1987). In the near-infrared, $\mathrm{HH} 240 \mathrm{~A}$ is detected in lines of [C I] and [S II]. In contrast, other lines dominated by emission from the proposed Mach disk, such as the [O I] (6300) and [N II]), require a shock speed of $200-300 \mathrm{~km} \mathrm{~s}^{-1}$. This gas cools slowly after the Mach disk shock, with a distance to cool down to $1000 \mathrm{~K}$ of $\sim 1.3 \times 10^{16} \mathrm{~cm}$ for a pre-shock (jet) density of $100 \mathrm{~cm}^{-3}$ (Hartigan et al. 1987). This is equal to the bow size. Therefore, the S II and $\mathrm{H} \alpha$ emission distributions should be quite extended, as observed. Optical spectroscopy should confirm this set up, in which a light and fast atomic jet pushes through a dense molecular medium. 
Figure 19 shows that weak optical [S II] line emission also arises from locations close to the $\mathrm{H}_{2}$ emission from $\mathrm{HH} 240 \mathrm{~B}$, C and D (Bohigas et al. 1993) (not only HH240A is visible in the optical). It is intriguing that optical emission is detectable from $\mathrm{HH} 240 \mathrm{C}$ given the $K$-band extinction of $1 \mathrm{mag}$, which implies a red band extinction of $A_{R} \sim 7$ mag (Rieke \& Lebofsky 1985). The optical emission appears curved, consistent with an alignment with the $\mathrm{H}_{2}$ bow. Note, however, that the images in Fig. 19 have been aligned according to the HH 240A peaks in the absence of precise spatial information. One interpretation is that the optical emission arises from a portion of the bow shock not subject to high red extinction. This could be consistent with our model for HH 240C in which the ambient medium is highly inhomogeneous with dense material possessing a small volume filling factor. The predicted column of gas through the bow, however, is not sufficient to provide the extinction. That is, the extinction should be caused by foreground L1634 cloud material.

It thus appears that the optical emission from HH 240B, C and $\mathrm{D}$ has still been detected despite quite high extinction. This is consistent with the non-detection of $1 \mu \mathrm{m}$ atomic lines in these objects (Nisini et al. 2002), suggesting that the intrinsic emission, even in the optical, is not strong and located at the bow shocks. These bows are probably not jet driven but are drifting along, still pushed by the decelerating remnants of jetswept clumps.

\section{Conclusions}

We have examined the structure of the $\mathrm{HH} 240 / \mathrm{HH} 241$ outflow in L1634. Images of the HH 240 bow shocks in $(1,0)$ and $(2,1)$ ro-vibrational $\mathrm{H}_{2}$ emission lines have revealed the excitation properties. Spectroscopy has provided detailed information on the bow shock dynamics.

To extract the physical and dynamical parameters, we have attempted to model the bows as steady-state curved shock fronts. We varied several parameters, including the bow physics, bow speed, bow shape, magnetic field and density.

We then compared our derived models with published data on $\mathrm{H}_{2}$ line fluxes, to determine column densities, and $\mathrm{CO}$ imaging and spectroscopy. From this vast body of data, we have reached several conclusions.

- The HH240 bow shocks propagate within an outflow medium with a radial projected speed of $-10 \mathrm{~km} \mathrm{~s}^{-1}$ relative to the cloud, implying an intrinsic outflow speed of $20 \mathrm{~km} \mathrm{~s}^{-1}$.

- The opposing bow shocks in HH 241 propagate within an outflow medium with a radial projected speed of $+10 \mathrm{~km} \mathrm{~s}^{-1}$ relative to the cloud, implying an intrinsic outflow speed of $20 \mathrm{~km} \mathrm{~s}^{-1}$.

- The CO outflow is associated with stationary cloud material i.e. no high radial speed is detected. Therefore, the gas set in motion, accelerated by just a few $\mathrm{km} \mathrm{s}^{-1}$ on both sides of the outflow, is molecular cloud material.

- HH240A corresponds very closely to a C-type bow. Several model parameters can be well constrained by the combined data sets including the orientation, bow velocity, density, ion fraction and intrinsic bow shape.

- A quite high magnetic field, as indicated by the Alfvén speed, implies that the material being shocked is the outflow itself and not undisturbed cloud material since the cloud would not be in virial equilibrium with the implied magnetic pressure. This is consistent with the pre-shock forward motion of the $\mathrm{H}_{2}$ gas.

- The $\mathrm{H}_{2}$ emission observed is associated with partly atomic gas. The distribution and excitation of shock-excited atomic gas suggests that a fast atomic jet impacts and drives along HH 240A.

- HH240C has higher vibrational excitation, higher preshock density and higher extinction than HH240A. Yet there is no associated $\mathrm{CO}$ outflow component. We therefore suggest that the $\mathrm{HH} 240 \mathrm{C}$ bow is now entering a denser region. While $\mathrm{H}_{2}$ emission is abruptly generated by the shock, sufficient $\mathrm{CO}$ gas has not yet accumulated. Furthermore, it is best modelled as a J-type bow undergoing transition into a $\mathrm{C}$-type bow as it enters the denser region.

The moderate molecular fractions suggest that the outflow has partly dissociated molecules in previous outburst episodes and molecules may have partly reformed in between episodes. The timescale for reformation at a density of $10^{4} \mathrm{~cm}^{-3}$, assuming the gas cools to $100 \mathrm{~K}$ is $\sim 1.6 \times 10^{5} \mathrm{yr}$. In comparison the outflow dynamical time is $\sim\left(0.42 \mathrm{pc} / 60 \mathrm{~km} \mathrm{~s}^{-1}\right)=7 \times 10^{3} \mathrm{yr}$. This implies that only a few per cent reformation may occur unless reformation occurs within dense clumps. More probably, most of the molecular gas in the outflow has not been previously dissociated but, instead, has passed through bow wings which sweep through most of the outflow without destroying the molecules.

The combination of our best-fit $70 \mathrm{~km} \mathrm{~s}^{-1}$ bow with a $20 \mathrm{~km} \mathrm{~s}^{-1}$ pre-shock outflow speed (given by the radial velocity data) yields a total speed of $90 \mathrm{~km} \mathrm{~s}^{-1}$ relative to the source. This is in excellent agreement with early proper motion studies of the optical emission associated with $\mathrm{HH} 240 \mathrm{~A}$ which yielded a proper motion of $90 \mathrm{~km} \mathrm{~s}^{-1}$, but probably with large errors in magnitude and direction.

The fractional ionisation we derive for the HH 240A C-type bow shock is $1 \times 10^{-5}$. This is well above the predicted ion level for cosmic-ray induced ionisation at the density of $2.5 \times 10^{3} \mathrm{~cm}^{-3}$ of $2 \times 10^{-7}$. We are, however, close to the densities appropriate for diffuse clouds in which external UV radiation penetrates and maintains a high fractional ionisation of the metals. We estimate a relationship of the form $\chi \sim$ $10^{-3} n^{-1 / 2} 10^{-0.5 A_{\mathrm{V}}}$ within the range of partially optically thick clouds exposed to the Galactic UV field. Given the low extinction of $A_{\mathrm{V}}=2-3$ found here and by Nisini et al. (2002), sufficient UV flux penetrates to maintain a fractional ionisation of order $10^{-5}$. The origin of the high ion fraction which has maintained HH $240 \mathrm{C}$ as a J-type shock $\left(\chi>10^{-4}\right)$ is not clear.

A global model for the outflow is suggested by the accumulated conclusions, as follows. Twin jets have driven out and pushed aside the background cloud. The jets are slowly precessing and have so produced a bipolar outflow which occupies two channels with mean outflow speeds of $20 \mathrm{~km} \mathrm{~s}^{-1}$. 
Furthermore, the jets are episodic, and so form and drive each bow shock for a limited time span after which the bow drifts until the associated momentum is drained. The question for theorists now is whether such an MHD model scenario can reproduce the observed spatial and velocity characteristics.

We can test our conclusions through (1) infrared predictions and (2) numerical simulations. Predicted infrared and farinfrared line luminosities are listed in Table 5. Many of the associated fluxes should be detectable from space-borne telescopes in the coming years.

Numerical simulations in three dimensions are necessary to simulate precessing jets. Spectroscopic predictions have been presented from such hydrodynamic simulations for atomic flows (Masciadri et al. 2002) and molecular flows (Smith et al. 1997; Völker et al. 1999). Based on the Smith et al. (1997) simulations, a jet-driven bow shock model for HH 240/241 was advanced by Lee et al. (2000). A new programme of molecular simulations is in progress (Rosen \& Smith 2003) within which we find strong support for the suggested global model (Rosen \& Smith, in preparation).

Acknowledgements. We thank Joaquin Bohigas for kindly retrieving the optical image, Brunella Nisini for providing us with detailed nearinfrared data, Alex Rosen for many discussions and the referee Roland Gredel for valuable criticisms. The UK Infrared Telescope is operated by the Joint Astronomy Centre on behalf of the UK Particle Physics and Astronomy Research Council. This research is supported by a grant to the Armagh Observatory from the Northern Ireland Department of Culture, Arts and Leisure and by the UK Particle and Astronomy Research Council (PPARC). We would like to acknowledge the data analysis facilities provided by the Starlink Project which is run by CCLRC/Rutherford Appleton Laboratory on behalf of PPARC.

\section{References}

Bachiller, R. 1996, ARA\&A, 34, 111

Bally, J., \& Lada, C. J. 1983, ApJ, 265, 824

Beltrán, M. T., Estalella, R., Ho, P. T. P., et al. 2002, ApJ, 565, 1069

Bohigas, J., Persi, P., \& Tapia, M. 1993, A\&A, 267, 168

Bohlin, R. C., Savage, B. D., \& Drake, J. F. 1978, ApJ, 224, 132
Bragg, S. L., Smith, W. H., \& Brault, J. W. 1982, ApJ, 263, 999

Brand, P. W. J. L., Moorhouse, A., Burton, M. G., et al. 1988, ApJ, 334, L103

Chernoff, D. F. 1987, ApJ, 312, 143

Cohen, M. 1980, AJ, 85, 29

Davis, C. J., Hodapp, K. W., \& Desroches, L. 2001, A\&A, 377, 285

Davis, C. J., Ray, T. P., Eisloeffel, J., \& Corcoran, D. 1997, A\&A, 324, 263

Davis, C. J., \& Smith, M. D. 1996, A\&A, 309, 929

De Vries, C. H., Narayanan, G., \& Snell, R. L. 2002, ApJ, 577, 798

Draine, B. T. 1980, ApJ, 241, 1021

Draine, B. T., Roberge, W. G., \& Dalgarno, A. 1983, ApJ, 264, 485

Hartigan, P., Raymond, J., \& Hartmann, L. 1987, ApJ, 316, 323

Hester, J. J., Stapelfeldt, K. R., \& Scowen, P. A. 1998, AJ, 116, 372

Hodapp, K., \& Ladd, E. F. 1995, ApJ, 453, 715

Jones, B. F., Cohen, M., Sirk, M., \& Jarrett, R. 1984, AJ, 89, 1404

Lee, C., Mundy, L. G., Reipurth, B., Ostriker, E. C., \& Stone, J. M. 2000, ApJ, 542, 925

Mardones, D., Myers, P. C., Tafalla, M., et al. 1997, ApJ, 489, 719

Masciadri, E., de Gouveia Dal Pino, E. M., Raga, A. C., \& Noriega-Crespo, A. 2002, ApJ, 580, 950

McKee, C. F., Storey, J. W. V., Watson, D. M., \& Green, S. 1982, ApJ, 259,647

Nisini, B., Caratti o Garatti, A., Giannini, T., \& Lorenzetti, D. 2002, A\&A, 393, 1035

Oliva, E., \& Origlia, L. 1992, A\&A, 254, 466

Reipurth, B., \& Bally, J. 2001, ARA\&A, 39, 403

Reipurth, B., Chini, R., Krugel, E., Kreysa, E., \& Sievers, A. 1993, A\&A, 273, 221

Richer, J. S., Shepherd, D. S., Cabrit, S., Bachiller, R., \& Churchwell, E. 2000, Protostars and Planets IV, 867

Rieke, G. H., \& Lebofsky, M. J. 1985, ApJ, 288, 618

Rosen, A., \& Smith, M. D. 2003, MNRAS, 343, 181

Smith, M. D. 1991, MNRAS, 252, 378

Smith, M. D. 1994, MNRAS, 266, 238

Smith, M. D., \& Brand, P. W. J. L. 1990, MNRAS, 245, 108

Smith, M. D., Froebrich, D., \& Eislöffel, J. 2003a, ApJ, 592, 245

Smith, M. D., Khanzadyan, T., \& Davis, C. J. 2003b, MNRAS, 339, 524

Smith, M. D., \& Mac Low, M.-M. 1997, A\&A, 326, 801

Smith, M. D., Suttner, G., \& Yorke, H. W. 1997, A\&A, 323, 223

Sugitani, K., Fukui, Y., \& Ogura, K. 1991, ApJS, 77, 59

Völker, R., Smith, M. D., Suttner, G., \& Yorke, H. W. 1999, A\&A, 343,953 